Historic, Archive Document

Do not assume content reflects current scientific knowledge, policies, or practices. 



\title{
Catalogue of Indian
}

\section{Insects}

\section{PART I-Acrydidae (Tettigidae)}

\author{
BY \\ T. BAINBRIGGE FLETCHER, \\ R.N, F.L.S., F.E.S., F.Z.S.,
}

(Imperial Entomologist.)

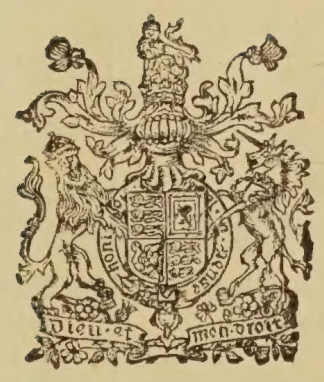

CALCƯTT̃A

SUPERINTENDENT GOVERNMENT PRINTING, INDIA 1921 


\section{Agents for the Sale of Books Published by the S uperintendent of Government Printing, India, Calcutta}

\section{IN EUROPE}

Constable \& Cc., 10, Orange Street, Leicester Square, London, W.C.

Kegan Paul, Trench, Trübner \& Co., 68-74, Carter Lane, E.C., and 39, New Oxford Street, Kondon, W.C.

Bernard Quaritch, 11, Grafton Street, New Bond Street, London, W.

R. B. King \& Sons, $2 \& 4$, Great Smith Street, Westminster, London, S.W.

H. S. King \& Co., 65, Cornhill, E.C., and 9, Pall Mall, London, W.

Grindlay \& Co., 54, Parliament Street, London, S.W.

Luzac \& Co., 46, Great Russell Street, London, W.C.

W. Thacker \& Co., 2, Creed Lane, London, E.C.

T. Fisher Unwin, Ltd., 1, Adelphi Terrace, London, W.C.

Wheldon \& Wesley, Ltd., 28, Essex street, London, W.C.

B. H. Blackwell, 50 \& 51, Broad Street, Oxford. Deighton Bell \& Co., Ltd., Cambridge.

Oliver and Boyd, Tweeddale Court, Edinburgh. E. Ponsonby, Ltd., 116, Grafton Street, Dublin. Ernest Leroux, 28, Rue Bonaparte, Paris.

Martinus Nijhoff, The Hague, Holland.

\section{IN INDIA AND CEYLON}

Thacker, Spink \& Co, Calcutta and Simla. Newman \& Co., Calcutta.

B. Cambray \& Co., Calcutta.

s. K. Lahiri \& Co., Calcutta.

B. Banerjee \& Co., Calcutta.

The Indian School Supply Depôt, 309, Bow Bazar Street, Calcutta, and 226, Nawabpur, Dacca.

Butterworth \& Co. (India), Ltd., Calcutta.

Rai M. C. Sarcar Bahadur and Sons, $90 / 2 A$, Harrison Road, Calcutta.

The Weldon Library, 57, Park Street, West, Calcutta.

Standard Literature Co., Limited, Calcutta.

Lal Chand \& Sons, Calcutta.

Association Press, Calcutta.

Higginbothams, \& Co., Madras.

V. Kalyanarama Iyer \& Co., Madras.

G. A. Natesan \& Co., Madras.

S. Murthy \& Co., Madras.

Thompson \& Co., Madras.

Temple \& Co., Madras.

P. R. Rama Iyer \& Co., Madras.

Vas \& Co.; Madras.

E. M. Gopalakrishna Kone, Madura.

Thacker \& Co., Ltd., Bombay.

D. B. Taraporevala, Sons \& Co., Bombay.

Mrs. Radhabai Atmairam Sagoon, Bombay.

Sunder Pandurang, Bombay.

Gopal Narayan \& Co., Bombay.

Ramchandra Govind \& Son, Kalbaderi, Bombay.
Proprietor, New Kitabkhana, Poona.

The Standard Bookstall, Karachi.

MIangaldas Harkisandas, Surat.

Karsandas Narandas \& Sons, Surat.

A. H. Wheeler \& Co., Allahabad, Calcutta and Bombay.

N. B. Mathur, Supt., Nazir Kanun-i-Hind Press, Allahabad.

Munshi Seeta Ram, Managing Proprietor, Indian Army Book Depôt, J uhi, Cawnpore.

Rai Sahib M. Gulab Singh \& Sons, Mufld-i-Am Press, Lahore and Aliahabad.

Rama Krishna \& Sons, Lahore.

Supt., American Baptist Mission Press, Rangoon.

Manager, the "Hitavada," Nagpur.

S. C. Talukdar, Proprietor, Students and Company, Cooch Behar.

A. M. \& F. Ferguson, Colombo, Ceylon.

Manager, Educational Book Depôts, Nagpur and Jubbulpore.*

Manager of the Imperial Book Depôt, 63 Chandney Chauk Street, Delhi.*

Manager, "The Agra Medical Hall and Onoperative Association, Ltd." (Successors to A. John \& Co., Agra).*

Supt., Basel Mission Book and Tract Depository, Mangalore.*

P. Varadachary \& Co., Madras.*

Ram Dayal Agarwala, 184, Katra, Allahabad.*

D. C. Anand \& Sons, Peshawar.*

Manager, Newal Kishore Press, Lucknow.* Maung Lu Gale, Proprietor, Law Book Dep 8t.

- Agents for the sale of Legislative Department publications only. 


\section{PREFACE.}

THE Acrydidæ constitute a group of the short-horned grasshoppers which is considered by many authors to be a sub-family of the Acrididæ, from which, however, the Acrydidæ are distinguished by (1) the fact that the claws of the tarsi are not provided with an arolium (or pad between the two terminal claws; also known as a pulvillus) and (2) the prolongation of the pronotum backwards over the abdomen. The first of the two distinctions is constant and serves to mark the Acrydidæ sharply from the Acrididæ, but the backward projection of the pronotum is found in some Acridids also. Walker (Ann. Ent. Soc. America XII 285 ; Dec. 1919) finds grounds for considering that the Acrydidx are more primitive than the Acrididx. On the whole, therefore, it seems preferable to rank the Acrydids as a distinct family.

The names under which these small grasshoppers are best known to entomologists have usually been variants of the names Tettix and Tetrix, both of which are synonyms of Acrydium, and, as Acrydium is the earliest described genus in this group, the family name is derivable from that of this genus.

The Acrydidæ are small to moderately small insects, usually most abundant in damp grassy localities. Many species of the subfamily Scelimeninæ are sub-aquatic, never found far from water and usually occurring on wet rocks alongside streams and pools, diving into and swimming beneath the water when disturbed, the hind tibiæ in many species being expanded and flattened to form swimming legs.

Little is on record regarding the life-histories and habits of these insects. The immature stages are often found in company with the adults on damp rocks, etc., where both appear to feed on moss and other low forms of vegetable life. One species has been found as a minor pest of cabbages at Pusa.

The literature on this group is fairly extensive, the more important publications on the Indian forms being :-

1834. Westwood, Zoological Journal, Vol. V.

1839. Serville, Hist. Nat. des Insectes, Orthoptères.

1842. de Haan, in Temminck's Verhandel., Orthoptera.

1861. de Saussure, "Etude sur quelques Orthoptères du musée de Genève" (Ann. Soc. Ent. France (4) I 469-494; 1860).

1871. Walker, Cat. of Dermaptera Saltatoria, Part V. 
1871. Stäl, Recensio Orthopterorum, Pars I.

1887. Bolivar, Essai sur les Acridiens de la tribu des Tettigidæ, (Ann. Soc. Ent. Belg. XXXI 175-313).

1893. Brunner, Révision du système des Orthopteres (Ann. Mus. Geriova XXXIII 103-114, t. 5.

1898. Bolivar, Contributions à l'étude des Acridiens. Espèces de la faune indo-et austro-malaisienne du Museo Civico di Storia Naturale di Genova (Ann. Mus. Genova XXXIX 66-101).

1902. Green, Entom. Monthly Mag. XXXVIII 215.

1902. Bolivar, Les Orthoptères de St. Joseph's College à Trichinopoly (sud de l'Inde) (Ann. Soc. Ent. France LXX 580-586).

1904. Hancock, The Tettigidæ of Ceylon (Spolia Zeylanica II 97157, 4 tabs.)

1906. Hancock, Wystman's Genera Insectorum, Tetriginæ.

1907. Hancock, Studies of the Tetriginæ in the Oxford University Museum (Trans. Entom. Soc. London, 1907, 213-244, t. 21).

1908. Hancock, A new Ceylonese Tettigid of the genus Eurymorphopus (Spol. Zeylan. V 113-114, fig .)

1909. Hancock, Further studies of the Tetriginæ in the Oxford University Museum (Trans. Ent. Soc. 1908, 387-426, t. 22).

1909. Bolivar, Nouvelles espèces d'Acridiens du Musée de Genève (Madrid, Bol. Soc. Españ. IX 393-408).

1909. Lefroy, Indian Insect Life, pp. 80-82.

1910. Hancock, Notes on Ceylonese Tetriginæ, with descriptions of some new species (Spol. Zeylan. VI 140-149, 2 figs.).

1910. Hancock, Third Paper on the Tetriginæ in the Oxford University Museum (Trans. Ent. Soc. 1910, 346-365, t. 49).

1910. Kirby, Synonymic Catalogue of Orthoptera, Vol. III.

1912. Hancock, Tetriginæ (Acridiinæ) in the Agricl. Research Institute, Pusa, with descriptions of new species (Ind. Agric. Ent. Mem. IV, No. 2, pp. 131-160).

1913. Hancock, Zoological Results of the Abor Expedition, Orthoptera I (Rec. Ind. Mus. VIII, 311-316, t. 15).

1914. Kirby, Fauna of British India, Orthoptera, Acridiidæ, pp. 11-80.

1915. Hancock, Indian Tetriginæ (Rec. Ind. Mus. XI 55-132, t. 14).

1918. Bolivar, Contribucion al conocimiento de la Fauna indica Orthoptera (Locustidae vel Acridiidce). (Revista de la Real Academia de Cieniras Exactas, Fisicas y Naturales de Madrid, Tomo XVI, numeros 6, 7, 8 \& 9 de la segunda serie). 
The following key to the sub-families of Indian Acrydidæ is adapted from Hancock (1915) :-

(Antennæ with all the articles excepting the basal and the small atrophied apical articles deplanate triquetrous, com-

$1\{$ posed of 8-10 joints; vertex transverse, very broad; or strongly acuminate produced . . . . . Tripetalocerinæ

Antennæ filiform . . . . . 2

Anterior íemcra above distinctly sulcate; pronotum anteriorly produced over the

$2\{$ head in a cornute process; antennæ

with 16-22 joints
Anterior femora more or less compressed

Carinate above . . . . 3

Frontal costa widely forked, the rami forming a frontal scutellum .

$3\{$ Frontal costa furcillate, but the rami diverge only moderately, or parallel, very frequently separated by a sulcus . 4

Pronotum anteriorly truncate, or rarely angulate produced; posterior angles of the lateral lobes of pronotum turned down, more or less rounded; first hindtarsal joint longer than third

Batrachidianæ

3

Cladonotinæ

Pronotum truncate anteriorly, posterior angles of lateral lobes laminate produced outwards, acute, or posteriorly obliquely truncate, rarely turned down

Posterior angles of lateral lobes of pronotum produced outwards, often spined ; first joint of posterior tarsi longer than third; posterior tibiæe strongly ampliate, or margins dilated toward the apices ; very frequently not or sparingly spinose . . . . .

Posterior angles of lateral lobes of proScelimeninæ notum little produced outwards, obliquely truncate behind, very rarely acute spinose ; first and third joints of hind tarsi nearly equal in length .

Acrydinæ 5

Metrodorinæ 
Keys to the genera will be found in the references quoted under each sub-family and to the species usually in the references quoted under each genus.

Pusa :

T. BAINBRIGGE FLETCHER.

25th March, 1921. 


\section{Catalogue of Indian Insects.}

\section{Part I.-ACRYDIDE (TETTIGIDE).}

\section{TRIPETALOCERINAE.}

Tripetalocerini, Kirby, F. I. Orth. p. 11 (1914) [key to genera]

Tripetalocerince, Hancock, Rec. Ind. Mus. XI 55-56 (1915) [key to genera].

\section{Tripetalocera, Westwood (1834).}

Type : ferruginea, Westw.

Westw., Zool. Journ. V 444 (1834); Bol., Ann.

S. E. Belg. XXXI 190, 196, 310 (1887);

Hanc., Gen. Ins. Tetr. p. 4 (1906) ; Kirby,

Cat. Orth. III, 1 (1910), F. I. Orth. p. 12

(1914) [redescr.]

ferruginea, Westw., Zool. Journ. V 444, 'Travancore ${ }^{5}$ t. 22 f. 3 (1834) $^{1}$; de Haan, Temm. Malabar ${ }^{4}$

Verh. Orth. pp. 166, 168, t. 22 f. "11 (1842) [Acridium (Tetrix) ferrugiPenang ${ }^{4}$ neum $]^{2}$; Bol., Ann. S. E. Belg. XXXI Borneo ${ }^{4}$ 310 , t. 5 f. 28 (1887) $^{3}$; Kirby, Cat. (eadem?) $)^{4}$ Orth. III $1(1910)^{4}$, F. I. Orth. pp. 1213, ff. $15,16(1914)^{5}$.

\section{Birmana, Brunner (1893).} Type : gracilis, Brunn.

Brunn., Ann. Mus. Genova XXXIII 113-114 (1893) ; Hanc., Gen. Ins. Tetr. p. 4 (1906) ; Kirby, Cat. Orth. III 1 (1910), F. I. Orth. pp. 13-14 (1914) ; Hanc., Rec. Ind. Mus. XV 61 (1915).

gracilis, Brunn., An Mus. Genova XXXIII 114 , t. 5 f. $47,(1893)^{1}$; Kirby, Cat. Bhamo ${ }^{1}, 4$ Orth III ${ }^{1}$ (1910), ${ }^{2}$ F. I. Orth p. 14 $(1914)^{3}$; Hanc, Rec. Ind. Mus. XI 61 $(1915)^{4}$. 


\section{CLADONOTINÆ.}

Cladonotini, Kirby, F. I. Orth. p. 14 (1914) [key to genera]

Cladonotince, Hanc., Rec. Ind. Mus. XI 56,

61 (1915) [key to genera].

\section{Fieberiana, Kirby (1914).}

Type : pachymerus, Fieb.

Plagiocephalus [nec Macq.], Fieber, Abh. Type: pachymerus, Fieb.

Königl.-böhm. Ges. Wiss. (5) III 407

(1845).

Fieberiana, Kirby, F. I. Orth. p. 14 (1914). Type : pachymerus Fieb. pachymerus, Fieber, Abh. Königl.-böhm. India ${ }^{3}$

Ges. Wiss. (5) III 407 (1845) [Plagiocephalus $]^{1}$; Kirby, Cat. Orth. III 7 (1910) [Piezotettix $]^{2}$, F. I. Orth. p. 15 (1914) [redescr. $]^{3}$

\section{Oxyphyllum, Hancock (1909).}

Type : pennatum, Hanc.

Hanc., T. E. S. 1908, 393 (1909), Rec. Ind.

Mus. XI 56, 61 (1915).

pennatum, Hanc., T. E. S. 1908, 393-394, Darjiling1 ${ }^{1}{ }^{2}$

t. 22 f. $3(1909)^{1}$, Rec. Ind. Mus. XI $61(1915)^{2}$

\section{Deltonotus, Hancock (1904).}

Type : subcucullatus, Wlk.

Precilotettix [nec Scudd.], Bol., Ann. S. E. Fr. Type : gibbiceps, Bol. LXX 580 (1902).

Deltonotus, Hanc., Spol. Zeyl. II 107, 111 Type: subcucullatus, (1904), Gen. Ins. Tetr. pp. 9, 14 (1906) ; Wlk.

Kirby, Cat. Orth. III 7 (1910), F. I.

Orth. p. 15 (1914) [redescr.]; Hanc., Rec. Ind. Mus. XI 56, 61 (1915).

gibbiceps, Bol., Ann. S. E. Fr. LXX 580 Madura1, 2,3 5 (1902) [Poecilotettix $]^{1}$; Kirby, Cat. Orth. III $7(1910)^{2}$, F. I. Orth. pp. 16-17 (1914) ${ }^{3}$; Hanc, Gen. Ins. Tetr. p. 14 $(1906)^{4}$, Rec. Ind. Mus. XI $61(1915)^{5}$. 
subcucullatus, Wik., Cat. Derm. Salt. Ceylon ${ }^{1,5}$ V 830 (1871) [Teltix subcucullata]1. Pundaluoya2, 4, 6

Deltonotus tectiformis, Hanc., Spol. Zeyl. Hantane ${ }^{2}$

II 107, 111, 154, t. 1 ff. 2, 2a (1904) $)^{2}$ Kandy ${ }^{7}$

Gen. Ins. Tetr. p. 14, t. 1 f. $1(1906)^{3}$,

T. E. S. $1907,215(1907)^{4}$.

Deltonotus subcullatus, Kirby, Cat. Orth. III $7(1910)^{5}$, F. I. Orth. pp. 1516, f. $17(1914)^{6}$.

Deltonotus subcullatus, Hanc., Rec.

Ind. Mus. XI $61(1915)^{?}$.

\section{Cladonotus, Saussure (1861).}

Type : humbertianus, Sauss.

Sauss., Ann. S. E. Fr. (4) I 478 (1861);

Bolivar, Ann. S. E. Belg. XXXI 184, 192, 208 (1887); Hancock, Spol. Zeyl. II 107, 112-113 (1904) [redescr.], Gen. Ins. Tetrig. pp. 10, 16 (1906) [charact.], T. E. S. 1908 394-395 (1909) [revised]; Kirby, Cat. Orth. III 9 (1910), F. I. Orth. p. 17 (1914) [redescr.]; Hanc., Rec. Ind. Mus. XI 56, 61-62 (1915) [key to spp.].

humbertianus, Sauss., Ann. S. E. Fr. (4) I Ceylon ${ }^{4}{ }^{6}$ 478 (1861)1; Bol., Ann. S. E. Belg. Peradeniya ${ }^{2,5}$ XXXI 209, t. 4 ff. 10, $10 w(1887)^{2}$; Trincomali ${ }^{2}, 5$. Hanc., Spol. Zeyl. II 107, 113-114 $(1904)^{3}$; Kirby, Cat. Orth. III 9 $(1910)^{4}$, F. I. Orth. pp. 17-18, f. 18 (1914)5; Hanc., Rec. Ind. Mus. XI $62(1915)^{6}$

latiramus, Hanc., Spol. Zeyl. II 107, 113, Ceylon ${ }^{3}, 5$ 114-116, t. 1 ff. 1, Ia (1904) ${ }^{1}$, Gen. Ins. Kandy ${ }^{1}{ }^{4}$, Tetrig. p. 16, t. 1 f. 3 (1906) ${ }^{2}$; Kirby, Cat. Orth. III $9(1910)^{3}$, F. I. Orth. p. 19 (1914) $)^{4}$; Hanc., Rec. Ind. Mus. XI $62(1915)^{5}$

turrifer, Wlk., Cat. Derm. Salt. V 843 Ceylon ${ }^{1-4}$ $(1871)^{1}$; Kirby, Cat. Orth. III 9 $(1910)^{2}$, F. I. Orth., pp. 18-19, f. 19 
(1914) ${ }^{3}$; Hanc., Rec. Ind. Mus. XI 62 $(1915)^{4}$.

\section{Tettilobus, Hancock (1909).}

Type : spinifrons, Hanc.

Teltilobus, Hanc., T. E. S. 1908396 (1909), Type : spinifrons, Hanc Rec. Ind. Mus. XI 56, 62 (1915).

Mnema, Kirby, F. I. Orth. p. 19 (1914).

pelops, Wlk., Cat. Derm. Salt. V 843 (1871) Ceylon ${ }^{1 \cdot 4}$

[Cladonotus] ${ }^{1}$; Kirby, Cat. Orth. III

9 (1910) [Cladonotus]'; Kirby, F. I.

Orth. p. 20, ff. 20, 21 (1914) [Mnema $]^{3}$;

Hanc., Rec. Ind. Mus. XI 62 (1915)

spinifrons, Hanc., T. E. S. 1908 396-397, Ceylon¹, 2

t. 22 f. $4(1909)^{1}$, Rec. Ind. Mus. XI

$62(1915)^{2}$.

\section{Gignotettix, Hancock (1909).}

Type : burri, Hanc.

Hanc., T. E. S. 1908 397-398 (1909), Rec.

Ind. Mus. XI 56, 62 (1915).

burri, Hanc., T. E. S. 1908 398, t. 22 f. 5 Pundaluoya ${ }^{1}$

$(1909)^{1}$, Rec. Ind. Mus. XI $62(1915)^{2}$.

\section{Potua, Bolivar (188\%).}

Type : coronata, Bol. [Borneo; Malacca].

Bol., Ann. S. E. Belg. XXXI 183, 192, 208

(1887) ; Hanc., Gen. Ins. Tetrig. pp. 10, 19

(1906) [charact.]; Kirby, Cat. Orth. III

11 (1910) ; Hanc., Rec. Ind. Mus. XI 56,

62 (1915) [charact.]

sabulosa, Hanc., Rec. Ind. Mus. XI 62-63 Satara distr. ${ }^{1}$ $(1915)^{\mathrm{I}}$.

\section{SCELIMENINE.}

Scelimenini, Kirby, F. I. Orth. pp. 20-21 (1914) [key to genera].

Scelimenince, Hanc., Rec. Ind. Mus. XI 56-58 [key to genera], 63 (1915). 


\section{Amphibotettix, Hancock (1906).}

Type : longipes, Hanc. [Borneo].

Hanc., Entl. News XVIII 86 (1906), Gen.

Ins. Tetrig. p. 22 (1906); Kirby, Cat. Orth.

III 12 (1910); Hanc., Rec. Ind. Mus. XI

57, 63 (1915).

rosaceus, Hanc., Rec. Ind. Mus. XI 63-64 Dawna Hills ${ }^{1}$ $(1915)^{1}$.

\section{Scelimena, Serville (1839).}

Type : producta, Serv. [Java].

Scelimena, Serv., Ins. Orth. p. 762 (1839); Type : producta, Serv.

Bol., Ann. S. E. Belg. XXXI 184,

193, 215 (1887) ; Hanc., Spol. Zeylan.

II 107, 116, 154 (1904) (redescr.], Gen.

Ins. Tetrig. pp. 21, 23 (1906) [charact.];

Kirby, Cat. Orth. III 12 (1910), F. I.

Orth. p. 21 (1914) [redescr.]; Hanc.,

Rec. Ind. Mus. XI 57, 64-65 (1915)

[charact.; key to Indian spp.]

Scelymena, Sauss., Ann. S. E. Fr. (4) I 484 Type: producta, Serv.

(1861).

Scelhymena, Bol., Ann. S. E. Fr. LXX 581 Type: harpago, Serv. (1902).

contracta, Wlk., Cat. Derm. Salt. V 841 India ${ }^{2}$

(1871) ${ }^{1}$; Kirby, Cat. Orth. III 13 (1910)

[Scelimena? ? $]^{2}$.

gavialis, Sauss., Ann. S. E. Fr. (4) I 485 Ceylon $^{5}$

(1861) $[\text { Scelymena }]^{1}$.

Peradeniya ${ }^{4}, 7$

Scelymena nodosa, Wlk., Cat. Derm. Pundaluoya ${ }^{4},{ }^{7},{ }^{9}$

Salt. V $840(1871)^{2}$.

Dambulla ${ }^{4}{ }^{7}$

Scelimena harpago [nec Serv.], Green, Ent. Maskeliya ${ }^{4}, 6,7$

Mo. Mag. XXXVIII 215 (1902) ${ }^{3}$.

Madulsima ${ }^{6}, 8$

Scelimena gavialis, Hanc., Spol. Zeyl. Haputale ${ }^{6}$

II $107,117-120, .154$, t. 1 ff. 4 , 4 a-c Labugama ${ }^{6}, 8$

(1904); ;irby, Cat. Orth. III 13 Kandy ${ }^{4}$

(1910)5; Hanc., Spol. Zeyl. VI 141-143 Pollibetta (Coorg).

(1910) [habits] ${ }^{6}$; Kirby, F. I. Orth.

pp. 24-25, f. 24 (1914) ${ }^{7}$; Hanc., Rec.

Ind. Mus. XI $66(1915)^{8}$, T. E. S. 1907,

218-219 (1907) . 
harpago, Serv., Ins. Orthopt. p. 763 (1839) Bombay ${ }^{1}, 4,5,7$ [Tetrix $]^{1}$. Matheran ${ }^{6}$

Tetrix harpago, de Haan, Temm., Verh., Bandra ${ }^{7}$ Orth. p. $166(1842)^{2}$. Igatpuri ${ }^{8}$

Scelimena harpago, Bol., Ann. S. E. Satara distr. ${ }^{8}$ Belg. XXXI 216-217, t. 4 f. 13 (1887) ${ }^{3}$. Kasara ${ }^{8}$ Scelhymena harpago, Bol., Ann. S. E. Fr. Almora? LXX 581 (1902). Coimbatore?

Scetimena harpago, Kirby, Cat. Orth. III 13 (1910) ${ }^{5}$; Hanc., Ind. Agric. Ceylon ${ }^{4}$

Ent. Mem. IV 132 (1912) ${ }^{6}$; Kirby,

F. I. Orth. pp. $23-24$, f. $23(1914)^{7}$; Hanc., Rec. Ind. Mus. XI 65-66 (1915) [variation]

logani, Hanc., Spol. Zeyl. II 107, 120-122, Ceylon ${ }^{3}$

t. 1 ff. 5, 5 a-c $(1904)^{1}$, Gen. Ins. Tetrig. Kandy ${ }^{1}, 5$

p. 24 , t. 2 f. 15 (1906) ${ }^{2}$; Kirby, Cat. Haragama ${ }^{1},{ }^{5}$

Orth. III 13 (1910) $)^{3}$ : Hanc., Spol. Pundaluoya ${ }^{5}$

Zeyl. VI. 143 (1910) [habits]'; Kirby, Madulsima ${ }^{4}$

F. I. Orth. pp. 25-26, f. 25 (1914) ; Kelawewa (N. C. P.) ${ }^{7}$

Hanc., Rec. Ind. Mus. XI 65 (1915)

[charact.] ${ }^{6}$, T. E. S. $1907218(1907)^{7}$;

Lefroy, Ind. Ins. Life, f. 21 on p. 76 $(1909)^{8}$.

\section{spinata, Hanc.}

Scelimena gavialis [nec Sauss.], Kirby, Trivandrum ${ }^{1,2}$

F. I. Orth. pp. 24-25 [part.] (1914)ㄴ․

Scelimena spinata, Hanc., Rec. Ind. Mus.

XI 66-67 (1915)².

uncinata, Serv., Orth. pp. $763-764$ (1839) Bombay $^{1}, 5$

$[\text { Tetrix }]^{1}$.

Scelymena contracta, Wlk., Cat. Derm. Salt. V $851(1871)^{2}$.

Scelimena uncinata, Bol., Ann. S. E. Belg. XXXI 216, $218(1887)^{3}$.

Scelimena? uncinata, Kirby, Cat. Orth. III $13(1910)^{4}$.

Scelimena uncinata, Kirby, F. I. Orth. p. 26, f. 26 (1914) ${ }^{5}$; Hanc., Rec. Ind. Mus. XI 65-66 (1915) [doubtful sp.] 
12. Eugavialidium, Hancock (1906).

Type : dentiumeris, Hanc. [Borneo].

Hanc., Gen. Ins. Tetrig., pp. 21, 24 (1906);

Kirby, Cat. Orth. III 14 (1910), F. I. Orth.

p. 30 (1914) [redescr.]; Hanc., Rec. Ind.

Mus. XI 57, 67-70 (1915) [key to spp.].

angulatum, Hanc., Rec. Ind. Mus. XI 73 Calcutta ${ }^{1}$ $(1915)^{1}$.

birmanicum, Brunn., Ann. Mus. Genova Burma ${ }^{3,5}$ XXXIII 104, t. 5 f. 37 (1893) [Gavia- Karen Hills ${ }^{1},{ }^{4}$ lidium]1.

Eugavialidium birmani, Hanc., Gen. Ins. Tetrig. p. $25(1906)^{2}$.

Eugavialidium birmanicum, Kirby, Cat. Orth. TII $14(1910)^{3}$.

Scelimena? birmanica, Kirby, F. I. Orth. p. $27(1914)^{4}$.

Eugavialidium birmanicum, Hanc., Rec. Ind. Mus. XI $73(1915)^{5}$.

\section{discale, Hanc.}

Scelimena producta [nec Serv.], Brunn., Karen Hills ${ }^{1}{ }^{2}$ Ann. Mus. Genova XXXIII 103 Sibsagar ${ }^{3}$ $(1893)^{1}$; Kirby, F. I. Orth. p. 22 [part.] $(1914)^{2}$.

Eugavialidum discalis, Hanc., Rec. Ind. Mus. XI $71(1915)^{3}$.

feæ, Bol., R. Soc. Españ. Nat. Hist. IX Karen Hills ${ }^{1},{ }^{2}$ 396 (1909)' ${ }^{1}$ Hanc., Rec. Ind. Mus. XI $73(1915)^{2}$.

flavopictum, Bol., Real. Soc. Españ. Nat. Calcutta1, 2 Hist. IX 394-395 (1909)'; Hanc., Rec.

Ind. Mus. XI $73(1915)^{2}$.

india, Hanc.

Scelimena india, Hanc., T. E. S. 1907 Cherrapunji ${ }^{1,3}$ 219 (1907) [male] ${ }^{1}$; Kirby, Cat. Orth. Assam 1, 2, 4 III $576(1910)^{2}$, F. I. Orth. p. 23 (1914) Shillong [orig. descr. reprod.] ${ }^{3}$

Eugavialidium indicum, Hanc., Rec. Ind. Mus. XI 72 (1915) [charact.] $]^{4}$ 
kempi, Hanc.

Scelimena india [nec Hanc. 1907], Hanc., Aborland ${ }^{1},{ }^{2}$ Rec. Ind. Mus. VIII 311 (1913) ${ }^{1}$.

Eugavialidium kempi, Hanc., Rec. Ind. Mus. XI 72-73 (1915)².

multidentatum, Hanc.

Scelimena producta [nec Serv.], Kirby, Dawna Hills', " F. I. Orth., p. 22 [part.] (1914) ${ }^{1}$.

Eugavialidium multidentatum, Hanc., Rec. Ind. Mus. XI 70-71 (1915) 2 .

saussurei, Hanc., Rec. Ind. Mus. XI 74 Calcutta ${ }^{1}$ $(1915)^{1}$.

\section{Abbasia, Kirby (1914).}

Type : subserrata, Kirby.

Kirby, F. I. Orth., pp. 27-28 (1914).

subserrata, Kirby, F. I. Orth. p. 28, f. 27 Tenmalai $^{1}$

$(1914)^{1}$.

[Travancore]

\section{Gavialidium, Saussure (1861).}

Type : crocodilus, Sauss.

Sauss., Ann. S. E. Fr. (4) I 481 (1861) ; Bol., Ann. S. E. Belg. XXXI 184, 193, 218 (1887); Hanc., Spol. Zeyl. II 107, 122 (1904) [redescr.], Gen. Ins. Tetrig. pp. 22, 25 (1906) [charact.]; Kirby, Cat. Orth. III 14 (1910), F. I. Orth. p. 28 (1914) [redescr.]; Hanc., Rec. Ind. Mus. XI 57 [charact.], 74 (1915).

alligator, Sauss., Ann. S. E. Fr. (4) I 483 Ceylon $^{1},{ }^{3}, 4$ (1861) [Scelymena] $]^{1}$; Bol., Ann. S. E. Kadugannawa ${ }^{5}$ Belg. XXXI 218, 219 (1887)2; Hanc., Spol. Zeyl. II 107, 122, 125 (1904) ${ }^{3}$; Kirby, Cat. Orth. III $15(1910)^{4}$, F. I. Orth. pp. 29-30 (1914) $)^{5}$ Hanc., Rec. Ind. Mus. XI 74 (1915) [probably immature crocodilus $]^{6}$. 
crocodilus, Sauss., Ann. S. E. Fr. (4) I 481 Ceylon $^{5}$

(1861) [Scelymena] ${ }^{1}$; Bol., Ann. S. E. Kadugannawa ${ }^{1},{ }^{3}, 7$

Belg. XXXI 218, 219 (1887) ${ }^{2}$; Hanc., Peradeniya ${ }^{8}$

Spol. Zeyl. II 107, 122, 123-124, t. 2 Pundaluoya ${ }^{3}$, 7, 8,9

ff. 11, $11 a, b .(1904)^{3}$, Gen. Ins. Tetrig. Haputale ${ }^{6}$

p. 25 , t. 2 ff. 16,16 a (1906) $)^{4}$; Kirby, Madulsima ${ }^{6}$.

Cat. Orth. III $14(1910)^{5}$; Hanc., Spol. Zeyl. VI 143-144 (1910) [habits]; Kirby, F. I. Orth. p. 29, f. $28(1914)^{7}$; Hanc., Rec. Ind. Mus. XI $74(1915)^{8}$, T. E. S. $1907220(1907)^{9}$.

\section{Bolotettix, Hancock (190\%).}

Type : validispinus, Hanc. [N. W. Borneo]. Hanc., T. E. S. 1907223 (1907); Kirby, Cat.

Orth. III 577 (1910); Hanc., Rec. Ind. Mus. XI 57 [charact.], 75 [key to Indian spp.] (1915).

anomalus, Hanc., Spol. Zeyl. VI 146 (1910) Madulsima 1.3 $^{\text {1-3 }}$

[Systolederus]1; Kirby, F. I. Orth. p. 46 (1914) [Systolederus $]^{2}$; Hanc., Rec. Ind. Mus. XI $76(1915)^{3}$.

armatus, Hanc., Rec. Ind. Mus. XI 76-77 Dawna Hills ${ }^{1}$ $(1915)^{1}$.

exsertus, Bol.

Criotettix exsertus, Bol., Ann. S. E. Fr. Kodaikanal1-4

LXX 583 (1902) ${ }^{\mathbf{1}}$; Kirby, Cat. Orth. III $16(1910)^{2}$, F. I. Orth. pp. 34-35, f. $33(1914)^{3}$.

Bolotettix exsertus, Hanc., Rec. Ind. Mus. XI $79(1915)^{4}$.

inermis, Hanc., Rec. Ind. Mus. XI 77-78 $(1915)^{1}$.

lobatus, Hanc.,

Systolederus lobatus, Hanc., Ind. Agric. Ent. Mem. IV 143-144 (1912)1.

Mazarredia lugubris, Kirby, F. I. Orth. p. 55, f. $51 \quad(1914)^{2}$.

Bolotettix lobatus, Hanc., Rec. Ind. Mus. XI 78-79 [redescr.] [1915] ${ }^{3}$. 
oculatus, Bol.,

Criotettix oculatus, Bol., Ann. Mus. Kodaikanal2, 4, 6

Genova XXXIX $71(1898)^{1}$, Ann. Coorg ${ }^{5}$

S. E. Fr. LXX $584(1902)^{2}$; Kirby,

Cat. Orth. III $16(1910)^{3}$, F. I. Orth. Java ${ }^{3},{ }^{4}, 6$

p. 34, f. 34 on p.37 (1914) ${ }^{4}$; Bol., Rev. Sumatra ${ }^{3},{ }^{4}, 6$

R. Acad. Cienc. Madrid (2) XVI 283 $(1918)^{5}$.

Bolotettix oculatus, Hanc., Rec. Ind. Mus. XI $76(1915)^{6}$.

pictipes, Hanc., Rec. Ind. Mus. XI 77 Shevaroys ${ }^{1}$ (1915) .

quadratus, Hanc., Rec. Ind. Mus. XI 79-80 $(1915)^{1}$.

triangularis, Hanc., Rec. Ind. Mus. XI 80 Sibsagar $^{1}$ $(1915)^{1}$.

16. Thoradonta, Hancock (1909).

Type : dentata, Hanc. [Penang].

Hanc., T. E. S. 1908407 (1909), Rec. Ind.

Mus. XI 57 [charact.], 80 (1915), T. E. S.

$1910358-359$ (1910).

apiculata, Hanc., Rec. Ind. Mus. XI 81-82 $(1915)^{1}$.

Singla (Darjiling distr.; $1,500$ feet $)^{1}$

Sikkim ${ }^{1}$

Sibsagar

Dawna Hills ${ }^{1}$

Tenasserim ${ }^{1}$

Sibsagar ${ }^{1}$

Singla (Darjiling distr.; $1,500$ feet $)^{1}$

sinuata, Hanc., Rec. Ind. Mus. XI 81 Satara distr. ${ }^{1}$ $(1915)^{1}$.

spiculoba, Hanc., Ind. Agric. Ent. Mem. IV Bihar ${ }^{1}$

$138(1912)^{1}$, Rec. Ind. Mus. VIII 312- Dibrugarh ${ }^{2}$

313 (1913) [variation] ${ }^{2}$, Rec. Ind. Mus. Calcutta ${ }^{3}$

XI $80-81 \quad(1915)^{3}$.

Rangoon ${ }^{3}$

Kandy ${ }^{3}$

17. Criotettix, Bolivar (188\%).

Type : tricarinatus, Bol.

Bol., Ann. S. E. Belg. XXXI 184, 193, 222

(1887); Hanc., Spol. Zeyl. II 108, 128

(1904) [redescr.], Gen. Ins. Tetrig. pp. 22, 
27, (1906) [charact.]; Kirby, Cat. Orth. III 16 (1910), F. I. Orth. p. 31 (1914) [redescr.]; Hanc., Rec. Ind. Mus. XI 58 [charact.], 82-84 [key to spp.] (1915).

æqualis, Hanc., Ind. Agric. Ent. Mem. IV Lebong1, 135-136 (1912) $)^{1}$, Rec. Ind. Mus. XI 87 , t. 14 f. $3(1915)^{2}$.

annandalei, Hanc., Rec. Ind. Mus. XI 87, Paresnath $(4,300 \mathrm{ft.})^{1}$ t. 14 f. $6(1915)^{1}$.

dohertyi, Hanc., Rec. Ind. Mus. XI 86-87, Upper Assam ${ }^{1}$ t. 14 f. $4(1915)^{1}$.

extremus, Hanc., Ind. Agric. Ent. Mem. Shevaroys ${ }^{1,2}$ IV 132-133 (1912) ${ }^{1}$, Rec. Ind. Mus. Nilgiris $(5,000 \mathrm{ft}$. XI 89, t. 14 f. 11 (1915) [possibly a form of maximus $]^{2}$.

fastiditus, Bol., Rev. R. Acad. Cienc. Taliparamba ${ }^{1}$ Madrid (2) XVI $284(1918)^{1}$.

flavopictus, Bol., Ann. S. E. Fr. LXX 582 Kodaikanal ${ }^{1},{ }^{3}$ (1902) ${ }^{1}$; Kirby, Cat. Orth. III 18 (1910) Anamalais ${ }^{4}$ [Acanthalobus] $]^{2}$, F. I. Orth. p. 41 Dawna Hills ${ }^{4}$ (1914) [Acanthalobus] ; Hanc., Rec. Cherrapunji ${ }^{5}$ Ind. Mus. XI 88, t. 14 f. $10(1915)^{4}$, T. E. S. $1907220(1907)^{5}$.

grandis, Hanc., Ind. Agric. Ent. Mem. IV 134-135 (1912) ${ }^{1}$, Rec. Ind. Mus. XI 89 , t. 14 f. $8(1915)^{2}$.

Chęrrapunji ${ }^{1},{ }^{2}$

Shillong

Khasis (3100 ft.)

Darjiling distr.(1,500ft. $)^{2}$

Shan Hills ${ }^{2}$

gravelyi, Hanc., Rec. Ind. Mus. XI 88, t. 14 f. $9(1915)^{1}$

indicus, Bol., Ann. S. E. Fr. LXX 581-582 (1902) ${ }^{1}$; Kirby, Cat. Orth. III 16 $(1910)^{2}$, F. I. Orth. pp. 33-34 (1914) ${ }^{3}$; Hanc., Rec. Ind. Mus. XI 85 (1915) Bol., Rev. R. Acad. Cienc. Madrid (2) XVI $283(1918)^{5}$.

Criotettix maculatus, Kirby, F. I. Orth. p. 32 , f. $30(1914)^{6}$.

maximus, Hanc., Rec. Ind. Mus. VIII 311- Aborland ${ }^{1}, 2$ 312 , t. 15 ff. 1, $1^{a}(1913)^{1}$, Rec. Ind. Janakmukh ${ }^{1}{ }^{2}$ Mus. XI 89, t. 14 f. $7(1915)^{2}$.

Ghumti (Darjiling distr.; $4,000 \mathrm{ft}.)^{2}$ 
montanus, Hanc., Ind. Agric. Ent. Mem. Simla ${ }^{1}{ }^{2}$ IV 133-134 (1912)1, Rec. Ind. Mus. XI 87, t. 14 f. $1(1915)^{2}$.

orientalis, Hanc., Rec. Ind. Mus. VIII 312, Dibrugarh1, 3 t. 15 ff. 4, $4^{a}(1913)^{1}$, Rec. Ind. Mus. XI 85, t. 14 f. $12(1915)^{2}$.

pallidus, Hanc., Rec. Ind. Mus. XI 85-86, Tenasserim ${ }^{1}$ t. 14 f. $5(1915)^{1}$.

rugosus, Bol., Ann. S. E. Belg. XXXI $228 \mathrm{Pegu}^{2}$ (1887) ${ }^{1}$; Brunn., Ann. Mus. Genova Rangoon ${ }^{3}$

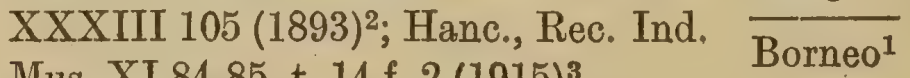
Mus. XI 84-85, t. 14 f. 2 (1915)

spinilobus, Hanc., Spol. Zeyl. II 108, 129131 , t. 3, ff. $12,12^{a},{ }^{b}(1904)^{1}$, Gen. Ins. Tetrig. p. 28, tab. f. $13(1906)^{2}$; Travancore Coast ${ }^{6}{ }^{7}$ Kirby, Cat. Orth. III $16(1910)^{3}$; Hanc., Spol. Zeyl. VI 145 (1910); Kirby, F. I. Orth. pp. 31-32 (1914) $)^{5}$; Hanc., Rec. Ind. Mus. XI 85, t. 14 f. 13 $(1915)^{6}$.

Criotettix obscurus, Kirby, F. I. Orth. pp. 32-33, f. 31 (1914)?

tricarinatus, Bol., Ann. S. E. Belg. XXXI Ceylon ${ }^{1}$, 184, 223, $224(1887)^{1}$, Ann. S. E. Fr. Weligama ${ }^{5}$ LXX 583 (1902) ${ }^{2}$; Hanc., Spol. Zeyl. Polgahawela ${ }^{5}$ II $108,128-129$, t. 3 ff. $15,15^{a, b}$ Kadugannawa ${ }^{3}$ ? (1904) ${ }^{3}$; Kirby, Cat. Orth. III 16 Peradeniya ${ }^{3}$, ?, 8 (1910)", Hanc., Spol. Zeyl. VI 144 Kandy ${ }^{3},{ }^{4}, 8$ (1910) [habits] $]^{5}$, Ind. Agric. Ent. Sigiriya ${ }^{8}$ Mem. IV . 135 (1912) $^{6}$; Kirby, F. I. Pundaluoya ${ }^{3}$, ? Orth. p. 33 f. 32 (1914) ${ }^{7}$; Hanc., Rec. Madulsima ${ }^{5}$ Ind. Mus. XI 85, t. 14 f. 14 (1915) ; Kodaikanal ${ }^{2}$ Bol., Rev. R. Acad. Cienc. Madrid (2) Shevaroys ${ }^{6}$. XVI $283(1918)^{9}$.

Coimbatore $^{9}$ Virajpet (Coorg)

\section{Loxilobus, Hancock (1904).}

Type : acutus, Hanc.

Hanc., Spol. Zeyl. II 108, 134 (1904), Gen. Ins. Tetrig. pp. 22, 29 (1906) [charact.]; Kirby, Cat. Orth. III 18 (1910), F. I. Orth. 
p. 41 (1914) [redescr.]; Hanc., Rec. Ind.

Mus. XI 58, 91 (1915) [charact.].

acutus, Hanc., Spol. Zeyl. II 108, 134--135, Pundaluoya ${ }^{1}{ }^{4}$

t. 1 f. 3, t. 3 ff. $16,16^{a, b}(1904)^{1}$, Gen. Ceylon ${ }^{2}, 3,5$

Ins. Tetrig. p. 29 , f: 14 , t. 2 f. 17

$(1906)^{2}$; Kirby, Cat. Orth. III 18

$(1910)^{3}$, F. I. Orth. p. $42(1914)^{4}$;

Hanc., Rec. Ind. Mus. XI $90(1915)^{5}$,

[nec Hanc., Ind. Agric. Ent. Mem.

IV 137 (1912), male,=parvispinus.]

assamus, Hanc., T. E. S. $1907223(1907)^{1}$;

Kirby, Cat. Orth. III $577(1910)^{2}$;

Cherrapunji ${ }^{1},{ }^{4}$

Hanc., Ind. Agric. Ent. Mem. IV 136

(1912)3; Kirby, F. I. Orth. p. 43

(1914) ${ }^{4}$; Hanc., Rec. Ind. Mus. XI 90

$(1915)^{5}$

\section{hancocki, Kirby,}

Loxilobus rugosus [nec Bol.], Hanc., Spol.

Zeyl. II 108, 134, 135-136, t. 3 ff. 17,

$17^{a, b}(1904)^{1}$, Gen. Ins. Tetrig. p. 30

$(1906)^{2}$, T. E. S. $1908401(1909)^{3}$,

Spol. Zeyl. VI $145(1910)^{4}$.

Loxilobus hancocki, Kirby, Cat. Orth. III $18(1910)^{5}$; Hanc., Ind. Agric. Ent. Mem. IV 137 (1912) ; Kirby, F. I. Orth., pp. 42-43, f. 40 (1914) ${ }^{7}$; Hanc., Rec. Ind. Mus. XI $90(1915)^{8}$.

parvispinus, Hanc., Rec. Ind. Mus. XI 90- Pusa ${ }^{1}{ }^{2}$

$91(1915)^{1}$.

Loxilobus acutus [nec Hanc. 1904], Hanc., Ind. Agric. Ent. Mem. IV 137 (1912) [male] ${ }^{2}$.

striatus, Hanc., Rec. Ind. Mus. XI 91 Calcutta ${ }^{1}$ $(1915)^{1}$.

Dawna Hills ${ }^{1}$

subulatus, Bol.,

Criotettix subulatus, Bol., Ann. S. E. E. Indies ${ }^{1-3}$

Belg. XXXI 185, 223, 227 (1887)'; Ceylon ${ }^{5}$

Kirby, Cat. Orth. III $16(1910)^{2}$, F. I.

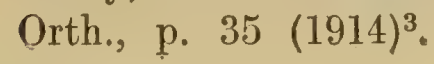


Loxilobus subulatus, Hanc., Rec. Ind. Mus. XI 90 (1915) ${ }^{4}$; Bol., Rev. R. Acad. Cienc. Ma rid (2) XVI 284 $(1918)^{5}$

vidali, Bol.,

Criotettix vidali, Bol., Ann. S. E. Belg. Karen Hills ${ }^{2}$, 5 XXXI 185, 223, 227 (1887)'; Brunn., Ann: Mus. Genova XXXIII 105 $(1893)^{2}$; Kirby, Cat. Orth. III 16 Philippines, ${ }^{\mathbf{1}}$ $(1910)^{3}$, F. I. Orth. pp. 35-36 (1914) ${ }^{4}$; Hanc., Rec. Ind. Mus. XI 89 (1915)5 Loxilobus vidali, Bol., Rev. R. Acad. Cienc. Madrid (2) XVI 284 (1918) ${ }^{6}$.

\section{Acanthalobus, Hancock (1904).}

Type : miliarius, Bol.

Hanc., Spol. Zeyl. II 108, 131 (1904), Gen. Ins. Tetrig. pp. 22, 28 (1906); Kirby, Cat. Orth. III 17 (1910), F. I. Orth. p. 36 (1914) [redescr.]; Hanc., Rec. Ind. Mus. XI 58 [charact.], 91-92 [key to spp.] (1915).

\section{bispinosus, Dalman,}

Acrydium bispinosum, Dalm., Vet. Acad. Burma ${ }^{8}$ Handl. 1818, p. $77^{1}$.

Karen Hills ${ }^{8}, 10$

Tettix bispinosa, Dalm., Anal. Entom. Sibsagar ${ }^{11}$ p. $86(1823)^{2}$.

Brahmaputra River

Tettix pallitarsis, Wlk., Cat. Derm. Salt. V 822-823 (1871) ${ }^{3}$.

Tettix armigera, Wlk., Cat. Derm. Salt. Penang ${ }^{12}$ V 826-827 (1871) ${ }^{4}$.

Tettix iatispina, Wlk., Cat. Derm. Salt. Java ${ }^{6}, ?$ V $837(1871)^{5}$. Sumatra ${ }^{10}$

Tettix bispinosus, Stål, Recens. Orth. I $150(1873)^{6}$.

Borneo $^{7}, 10$

Criotettix bispinosus, Bol., Ann. S. E. Hongkong ${ }^{3}, 4$

Belg. XXXI 185, 223, $226(1887)^{7}$; China ${ }^{3}, 6,7,9,10$

Brunn., Ann. Mụs, Genova XXXIII $105(1893)^{8}$. 
Acanthalobus bispinosus, Kirby, Cat.

Orth. III 17-18 $(1910)^{9}$, F. I. Orth. pp. 37-38 (1914) ${ }^{10}$; Hanc., Rec. Ind. Mus. XI 93-94 (1915) ${ }^{11}$, T. E. S. 1907, $222(1907)^{12}$.

cuneatus, Hanc., Spol. Zeyl. II $108(1904)^{1}$, Colombo ${ }^{2}{ }^{4}$ Spol. Zeyl. II 133-134 (1904) [A. miliarius cuneatus]; Kirby, Cat. Orth. III $17(1910)^{3}$, F. I. Orth. pp. 40-41, ff. 37-39 (1914) $)^{4}$; Hanc., Rec. Ind. Mus. XI 92 (1915) [apparently shortwing form of miliarius $]^{5}$.

curticornis, Hanc., Rec. Ind. Mus. XI 92- Satara distr. ${ }^{1}$ $93(1915)^{1}$.

\section{inornatus, Wlk.,}

Tettix inornata, Wlk., Cat. Derm. Salt. Burma ${ }^{5}$ V $834(1871)^{1}$.

Criotettix saginatus. Bol., Ann. S. E. Belg. Rangoon ${ }^{3}$, ", ? XXXI 185，223，225-226 (1887) ${ }^{2}$; Madura ${ }^{4}$

Brunn., Ann. Mus. Genova XXXIII 104, t. 5 f. 38 (1893); Bol., Ann. S. E.

Fr. LXX 583 (1902)

Acanthalobus inornatus, Kirby, Cat. Orth.

III $17(1910)^{5}$, F. I. Orth. pp. 39-40, f. 36 (1914). ${ }^{6}$

Acanthalobus saginatus, Hanc., Rec. Ind. Java ${ }^{2},{ }^{5}, 6$ il

Mus. XI $93(1915)^{7}$, T. E. S. 1907, 221 Sumatra $(1907)^{7 A}$. China $^{2 \mathrm{~A}}$

Acanthalobus inornatus, Bol., Rev. R. Acad. Cienc. Madrid (2) XVI 285 (1918) [doubts whether inornatus and saginatus are really only one sp. $]^{8}$.

miliarius, Bol., Ann. S. E. Belg. XXXI 185, Ceylon ${ }^{1}, 4$ 223, 226 (1887) [Criotettix] ${ }^{1}$; Hanc., Colombo ${ }^{2},{ }^{5}{ }^{7}$ Spol. Zeyl. II 108, 131, 132-133, t. 2 Galle ${ }^{5}$ ff. $8,8^{a}(1904)^{2}$, Gen. Ins. Tetrig. p. 29, Trincomali ${ }^{7}$ t. 2, ff. 19, $19^{7}(1906)^{3}$; Kirby, Cat. Kandy ${ }^{2}{ }^{6}$ Orth. III $17(1910)^{4}$; Hanc., Spol. Zeyl. Peradeniya ${ }^{2}, 6$ VI $145(1910)^{5}$; Kirby, F. I. Orth. Pundaluoya ${ }^{8}$ p. 37, [nec f. 34] (1914) ${ }^{6}$; Hanc, i ec. 
Ind. Mus. XI $92(1915)^{7}$, T. E. S. 1907 $222(1907)^{8}$.

rufescens, Kirby, F. I. Orth. pp. 38-39, Gangtok ${ }^{1}$ f. $35(1914){ }^{1}$

Kurseong ${ }^{1}$

Calcutta ${ }^{1}$

20. Tettitelum, Hancock (1915).

Type: hastulatum, Kirby.

Tettitelum, Hanc., Rec. Ind. Mus. XI 94 (1915).

Tettitellum, Hanc., Rec. Ind. Mus. XI 58 (1915) [error typogr.].

hastulatum, Kirby, F. I. Orth., p. 30, Tenasserim 1,2 f. 29 (1914) [Eugavialidium] ${ }^{1}$.

Tettitelum hastatum, Hanc., Rec. Ind. Mus. XI 94-95 (1915)².

\section{METRODORINAE.}

Metrodorini, Kirby, F. I. Orth. pp. 43-44 (1914) [key to genera].

Metrodorino, Hanc., Rec. Ind. Mus. XI 58-59 [charact. ; key to genera], 95 (1915).

21. Systolederus, Bolivar (1887).

Type : haani, Bol. [Philippines].

Bol., Ann. S. E. Belg. XXXI 185, 194, 234 (1887) ; Hanc., Spol. Zeyl. II 108, 136 (1904) [redescr.], Gen. Ins. Tetrig. pp. 30, 33 (1906) [charact.]; Kirby, Cat. Orth. III 20 (1910), F. I. Orth. p. 44 (1914) [redescr.]; Hanc., Rec. Ind. Mus. XI 58 [charact.], 95 (1915).

cinereus, Brunner, Ann. Mus. Genova Karen Hills. ${ }^{1-4}$ XXXIII 105 (1893)'; Kirby, Cat. Orth. III $20(1910)^{2}$, F. I. Orth. pp. 4445, f. 41 (1914) ${ }^{3}$; Hanc., Rec. Ind, Mus. XI 95 (1915) ${ }^{4}$. 
greeni, Bol., Ann. S. E. Fr. LXX 584 Pundaluoya ${ }^{1}, 2,6,7$

(1902)1; Hanc., Spol. Zeyl. II 108, 137- Madulsima ${ }^{5}$, ?

138, t. 2 ff. $9,9^{a, b}(1904)^{2}$, Gen. Ins. Haputale ${ }^{5}$

Tetrig. p. 34, t. 2 f. 14 (1906) ${ }^{3}$; Kirby, Maskeliya ${ }^{5},{ }^{7}$

Cat. Orth. III $20(1910)^{4}$; Hanc., Spol. Kadugannawa ${ }^{2}, 6$

Zeyl. VI 145-146 (1910) [habits] ${ }^{5}$; Kodaikanal ${ }^{1,}{ }^{6}$

Kirby, F. I. Orth. p. 45, f. $42(1914)^{6}$; Nilgiris?

Hanc., Rec. Ind. Mus. XI 95 (1915)? . Virajpet (Coorg)

Pusa

\section{Hancockia, Kirby (1914).}

Type : portentosa, Kirby.

Kirby, F. I. Orth. p. 46 (1914).

portentosa, Kirby, F. I. Orth. pp. 46-47, Travancore ${ }^{1}$

f. $43(1914)^{1}$.

\section{Eurymorphopus, Hancock (1906).}

Type : cunctatus, Bol. [New Caledonia].

Hanc., Gen. Ins. Tetrig. pp. 30, 35 (1906);

Kirby, Cat. Orth. III 21 (1910), F. I.

Orth. pp. 48-49 (1914) (redescr.]; Hanc.,

Rec. Ind. Mus. XI 58 [charact.], 95 (1915).

latilobus, Hanc., Spol. Zeyl. V 113-114, Undugodata

f. $1(1908)^{1}$; Kirby, F. I. Orth. p. 49, Kandy ${ }^{3}$

ff. $46,47(1910)^{2}$; Hanc., Rec. Ind.

Mus. XI $95(1915)^{3}$.

\section{Spadotettix, Hancock (1910)}

Type: fetcheri, Hanc.

Hanc., Spol. Zeyl. VI 146-147 (1910), Ind. Agric. Ent. Mem. IV 141-142 (1912); Kirby, F. I. Orth. p. 70 (1914) [redescr.]; Hanc., Rec. Ind. Mus. XI 58 [charact.], 95 (1915).

fletcheri, Hanc., Spol. Zeyl. VI 147-148, Madulsima'. figs. $(1910)^{1}$; Kirby, F. I. Orth. p. 71 (1914) ${ }^{2}$; Hanc., Rec. Ind. Mus. XI $95(1915)^{3}$. 
provertex, Hanc., Ind. Agric. Ent. Mem. Shevaroys ${ }^{1}$

IV 142-143 (1912)1 ${ }^{1}$, Rec. Ind. Mus.

XI $95(1915)^{2}$.

\section{Apterotettix, Hancock (1904).}

Type : oblusus, Hanc.

Hanc., Spol. Zeyl. II 108, 140 (1904), Gen.

Ins. Tetrig. pp. 30, 35 (1906) [charact.];

Kirby, Cat. Orth. III 21 (1910), F. I. Orth.

p. 47 (1914) [redescr.]; Hanc., Rec. Ind.

Mus. XI 58 [charact.], 95 (1915).

obtusus, Hanc., Spol. Zeyl. II 108, 140- Pundaluoya', 4

141, t. 3 ff. $13,13^{4, b}$ (1904)1, Gen. Ins. Peradeniya ${ }^{1}, 4,5$

Tetrig. p. 35, f. $16(1906)^{2}$; Kirby,

Cat. Orth. III $21(1910)^{3}$, F. I. Orth.

p. 48, ff. 44, 45 (1914) ; Hanc., Rec.

Ind. Mus. XI 96 (1915)5.

\section{Amphinotus, Hancock (1915).}

Type: pygmaeus, Hanc.

Hanc., Rec. Ind. Mus. XI 58-59, 96 (1915).

pygmæus, Hanc., Rec. Ind. Mus. XI 96$97(1915)^{1}$.

Hakgala!

Pundaluoya ${ }^{1}$

\section{Mazarredia, Bolivar (188\%).}

Type : gemella, Bol. [Philippines].

Bol., Ann. S. E. Belg. XXXI 185, 194, 236237 (1887) ; Brunn., Ann. Mus. Genova XXXIII 106 (1893); Hanc., Spol. Zeyl. II 108, 138 (1904) [redescr.], Gen. Ins. Tetrig. pp. 32, 42 (1906) [charact.] ; Kirby, Cat. Orth. III 24 (1910), F. I. Orth. pp. 4851 (1914) [redescr.] ; Hanc., Rec. Ind. Mus. XI 59 [charact.], 97-99 [key to spp.] (1915). convergens, Brunn., Ann. Mus. Genova XXXIII 106, 107-108, t. 5 f. 40 $(1893)^{1}$; Kirby, Cat. Orth. III 26 $(1910)^{2}$, F. I. Orth. p. $52(1914)^{3}$; Hanc., Rec. Ind. Mus. XI 101 (1915)

Karen Hills ${ }^{1}, 3$ Tenasserim ${ }^{1},{ }^{3}$ 
cristulata, Bol., Ann. S. E. Fr. LXX 584- Madura ${ }^{1,3}$ $585(1902)^{1}$; Kirby, Cat. Orth. III 25 $(1910)^{2}$, F. I. Orth. pp. 52-53 (1914) $)^{3}$; Hanc., Rec. Ind. Mus. XI 102 $(1915)^{4}$.

dubia, Hanc., Ind. Agric. Ent. Mem. IV Lebong1, 2 141 (1912), Rec. Ind. Mus. XI 101 Cherrapunji $(1915)^{2}$.

ghumtiana, Hanc., Rec. Ind. Mus. XI $100-101(1915)^{1}$.

inæqualis, Brunn., Ann. Mus. Genova Kirby, Cat. Orth. III $25(1910)^{2}$, F. I. Orth. p. 53, f. 49 (1914) ${ }^{3}$; Hanc., Rec. Ind. Mus. XI 99 (1915) ${ }^{4}$.

insignis, Kirby, F. I. Orth. p. 54, f. 50 $(1914)^{1}$.

insularis, Bol., Ann. S. E. Belg. XXXI 237, 239 (1887) ${ }^{1}$; Hanc., Spol. Zeyl. II $108,138-140$, t. 2 ff. $7,7^{a, b}(1904)^{2}$, T. E. S. $1907230(1907)^{3}$; Kirby, Cat. Orth. III $25(1910)^{4}$, F. I. Orth. p. 52 $(1914)^{5}$; Hanc., Rec. Ind. Mus. XI $102(1915)^{6}$.

laticeps, Bol., Bol. Soc. Españ. IX 399 (1909) ${ }^{1}$; Hanc., Rec. Ind. Mus. XI 102 $(1915)^{2}$.

latifrons, Hanc., Ind. Agric. Ent. Mem. IV 139 (1912) ${ }^{1}$, Rec. Ind. Mus. XI 102 $(1915)^{2}$.

lativertex, Brunn., Ann. Mus. Genova XXXIII 106, 108, t. 5 f. $41(1893)^{1}$; Kirby, Cat. Orth. III $26(1910)^{2}$, F. I. Orth. p. 54 (1914) ${ }^{3}$; Hanc., Rec. Ind. Mus. XI $100(1915)^{4}$

ophthalmica, Bol., Bol. Soc. Españ. IX Sibsagar ${ }^{1}{ }^{2}$ 399 (1909) ${ }^{1}$; Hanc., Rec. Ind. Mus. XI $102(1915)^{2}$

perplexa, Hanc., Rec. Ind. Mus. XI 101 Sikkim $^{1}$ $(1915)^{1}$ 
sculpta, Bol., Ann. S. E. Belg. XXXI 237, $238(1887)^{1}$; Brunn., Ann. Mus. Genova XXXIII $107(1893)^{2}$; Hanc., T. E. S. 1908405 (1909) $^{3}$; Kirby, Cat. Orth. III $25(1910)^{4}$, F. I. Orth. p. 51 [nec f. 48] (1914) ${ }^{5}$; Hanc., Rec. Ind. Mus. XI 100 (1915) ${ }^{6}$; Boliv., Rev. R. Acad. Cienc. Madrid (2) XVI 285 (1918)?

sikkimensis, Bol., Bol. Soc. Españ. IX Sikkim² 398-399 (1909)'; Hanc., Rec. Ind. Mus. XI $100(1915)^{2}$.

singlaënsis, Hanc., Rec. Ind. Mus. XI Singla (Darjiling distr.; 99-100 $(1915)^{1}$.

\section{Xistra, Bolivar (188\%).}

Type : gogorz $\infty$, Bol. [Philippines].

Bol., Ann. S. E. Belg. XXXI 186, 194, 242 (1887); Brunn., Ann. Mus. Genova XXXIII 108 (1893); Hanc., Gen. Ins. Tetrig. pp. 32, 45 (1906) [charact.]; Kirby, Cat. Orth. III 27 (1910), F. I. Orth. p. 55 (1914) [redescr.]; Hanc., Rec. Ind. Mus. XI 59 [charact.], 102 (1915).

\section{dubia, Brunn.,}

Xistra? dubia, Brunn., Ann. Mus. Karen Hills ${ }^{1},{ }^{3}$ Genova XXXIII 108-109, t. 5 f. 42 (1893)1; Kirby, Cat. Orth. III 27 $(1910)^{2}$, F. I. Orth. p. $56(1914)^{3}$.

Xistra dubia, Hanc., Rec. Ind. Mus. XI $102(1915)^{4}$.

sikkimensis, Hanc., Rec. Ind. Mus. XI Sikkim ${ }^{1}$ 102-103 (1915) ${ }^{1}$.

stylata, Hanc., T. E. S. 1907 231-232 Puttalam ${ }^{1}, 4$ (1907)1, T. E. S. 1908409 (1909)2; Kandy² Kirby, Cat. Orth. III $577(1910)^{3}$, Nagody (S. Kanara) ${ }^{6}$ F. I. Orth. p. 56 (1914) $^{4}$; Hanc., Rec. Coorg Ind. Mus. XI 102 (1915) ; Boliv., Rev. R. Acad. Cienc. Madrid (2) XVI 286 $(1918)^{6}$.
"East Indies"1

Tenasserim ${ }^{2}, 4,5$

Karen Hills ${ }^{2}, 5$

Assam ${ }^{3}$

Sukna ${ }^{6}$

$1,500$ feet $)^{1}$ 


\section{Xistrella, Bolivar (1909).}

Typo: Aromadarier, Bubl.

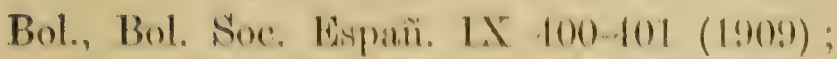

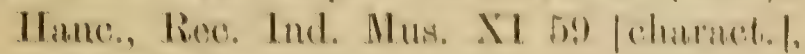
$103(1915)$.

dromadaria, Bol., Bol. Soo. Mispari. IX Nilikim!, 401 (1909)'; Hime., liece. Ind. Mluis.

XI 103 (1915) | fommlo deser. $\left.\right|^{3}$

\section{Lamellitettix, Hancock (1904).}

'lypo: aculus, Hinte.

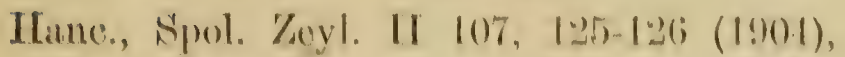

Gou. Ins. 'Totrig. Pr. 31, 11 (19)(i)

[eharact.]; Kirby, (at. ()rih. III 21

(1910), 1. 1. Orth. P. 19) (19)1.1) Lrodeser.l;

Ilane, Rec. Ind. Mus. XI b!) [eharact.],

$103(19) 5)$.

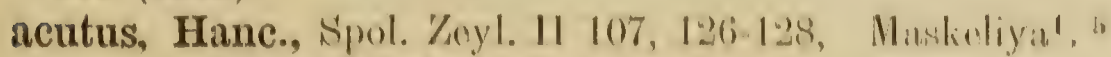

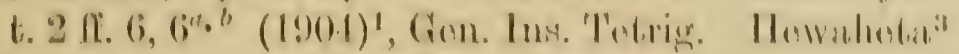

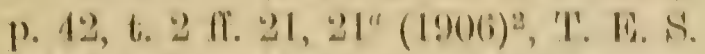

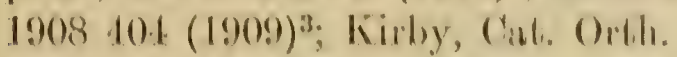

III 24 $(1910)^{4}$, H. I. Oilh. \%. 50

$(1914)^{5}$.

fleteheri, Hanc., lioe. Ind. Mus. XI L03 Anmmalais $(1915)^{1}$.

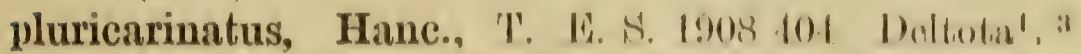

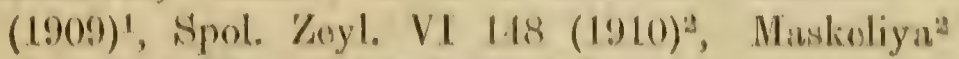
Rec. Ind. Mus. XI $103(1915)^{3}$.

\section{Hyboella, Hancock (1915)。}

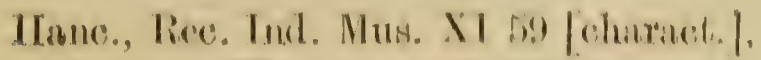
10.1-109 LKo.y 60 spl). | (19)1.5).

'Туре: : conlala, Hunc.

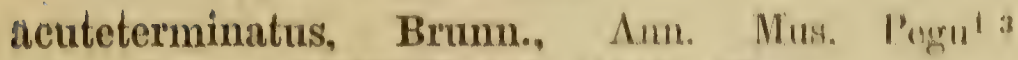

Genova XXXIII II' (1893) |(7opilo-

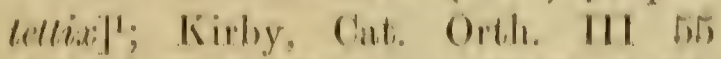

$(19) 10)^{2}$, 14. 1. Orlh. 19. 75i-76, 1. 70

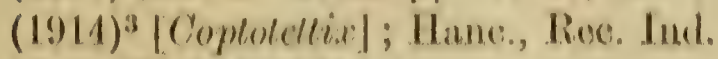

Mus. XI $106(1915)^{4}$. 
angulifrons, Hanc., Rec. Ind. Mus. XI Dawna Hills ${ }^{1}$ 108-109 $(1915)^{1}$.

conioptica, Hanc., Rec. Ind. Mus. XI 109 Singla (Darjiling distr.; $(1915)^{1}$. $1,500$ feet $)^{1}$ Assam ${ }^{1}$

\section{dilatata, de Haan,;}

Acridium (Tetrix) dilatatum, de Haan, Kare Hills ${ }^{3}, 5$ Temm., Verh. Orth. pp. 167, 169, t. 22 f. $12(1842)^{1}$.

Tettix dilatatus, Bol., Ann. S. E. Belg. XXXI 187, 257, 260 (1887)2; Brunn., Java1 1 2, 4, 5 Ann. Mus. Genova XXXIII 109 $(1893)^{3}$.

Acrydium dilatatum, Kirby, Cat. Orth. III $45(1910)^{4}$, F. I. Orth. p. 68 $(1914)^{5}$.

Hyboella dilatata, Hanc., Rec. Ind. Mus. XI $107(1915)^{6}$.

\section{latifrons, Brunn.,}

Coptotettix latifrons, Brunn., Ann. Mus. Pegu1, 3 Genova XXXIII 112, t. 5 f. 44 (1893)1; Pusa ${ }^{3}$

Kirby, Cat. Orth. III $55(1910)^{2}$, F. I. Orth. pp. 74-75, ff. 67-69 (1914)

Hyboella latifrons, Hanc., Rec. Ind. Mus. XI $106(1915)^{4}$.

nullipennis, Hanc., Rec. Ind. Mus. VIII Janakmukh ${ }^{1,2}$

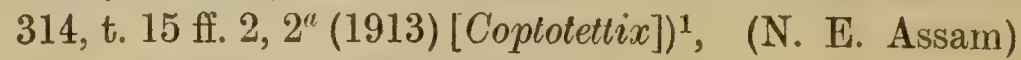
Rec Mus. XI 106 (1915) ${ }^{2}$.

obest, Hanc., Rec. Ind. Mus. XI 107-108 Ghumti (Darjiling distr.; $(1915)^{1}$. $4,000$ feet $)^{1}$

problematica, Bol.,

Coptotetix problematicus, Bol., Real Soc. Upper Assam ${ }^{1}, 2$ Españ. IX 401 (1909)'1.

Hyboella problematica, Hanc., Rec. Ind. Mus. XI 109 (1915)2.

tentata, Hanc., Rec. Ind. Mus. XI 106- Sibsagar ${ }^{1}$ $107(1915)^{1}$. 


\section{tumida, Hanc.,}

Coptoteltix tumidus, Hanc., Rec. Ind. Dibrugarh ${ }^{1,2}$

Mus. VIII 313-314, t. 15 ff. $3,3^{\text {t }}$ $(1913)^{1}$.

Hyboella tumida, Hanc., Rec. Ind. Mus. XI $106(1915)^{2}$.

\section{ACRYDINÆ 。}

Acrydiini, Kirby, F. I. Orth. p. 57 (1914) [key to genera].

Tettigince, Hanc., Rec. Ind. Mus. XI 59-60 [charact.; key to genera], 109 (1915).

\section{Teredorus, Hancock (1906).}

\section{Type: stenofrons, Hanc. [Peru]}

Teredorus, Hanc., Gen. Ins. Tetrig. pp. 51-52 (1906); Kirby, Cat. Orth. III 30 (1910); Hanc., Rec. Ind. Mus. XI 109-110 (1915).

Terederus [error typogr.], Hanc., Rec. Ind. Mus. XI 59 [charact.] (1915).

carmichaeli, Hanc., Rec. Ind. Mus. XI Singla (Darjiling distr.; $110(1915)^{1}$. $1,500$ feet $)^{1}$

frontalis, Hanc., Rec. Ind. Mus. XI 110 Simla Hills $(5,000 \mathrm{ft} .)^{1}$ $(1915)^{1}$.

\section{Paratettix, Bolivar (188\%).}

Type : meridionalis, Rambur [Mediterranean Region].

Batrachidia [nec Serv.], Sauss., Rev. Zool. (2) XIII 401 (1861).

Paratettix, Bol., Ann. S. E. Belg. XXXI 187, 195, 270 (1887); Finot, Faune France, Orth. pp. 100, 169 (1889); Lugger, Orthopt. Minnesota pp. 196, 199 (1897); Morse, Biol. Centr. Am., Orth. II 5, 11 (1900) ; Hanc., Tettig. N. Amer. pp. 36, 39, 108 (1902); Jacobs. and Bianchi, Prem. i Lozhn. Ross. Imp. pp. 164, 175, 211 
(1902); Blatchley, Rep. Indiana Dep. Geology XXVII 218, 227 (1903); Hanc., Spol. Zeyl. II 108, 110, 144 (1904) [redescr.], Gen. Ins. Tetrig. pp. 52, 55 (1906) [charact.]; Kirby, Cat. Orth. III 32 (1910); Hanc., Ind. Agric. Ent. Mem. IV 149 (1912); Kirby, F. I. Orth. pp. 60-61 (1914) [redescr.]; Hanc., Rec. Ind. Mus. XI 60 [charact.], 111-112 [key to spp.] (1915).

alatus, Hanc., Rec. Ind. Mus. XI 113-114 Ghumti (Darjiling distr.; $(1915)^{1}$. $4,000$ feet $)^{1}$

curtipennis, Hanc., Ind. Agric. Ent. Lebong1

Mem. IV 146-147 (1912) [Coptoteltix] ${ }^{1}$, Darjiling distr. (4,000

Rec. Ind. Mus. XI $113(1915)^{2}$.

dorsifer, Wlk. feet) ${ }^{2}$

Upper Shillong $(6,000$ feet)

Tettix dorsifera, Wik., Cat. Derm. Salt. Bombay ${ }^{1-3}$ V $825(1871)^{1}$.

Acrydium dorsiferum, Kirby, Cat. Orth. III $45(1910)^{2}$.

Paratettix? dorsifer, Kirby, F. I. Orth. p. 63, f. $55(1914)^{3}$.

hirsutus, Brunn., Ann. Mus. Genova Teinzo1, 3 XXXIII 110, t. 5 ff. $43^{a, b}$ (1893) ${ }^{1}$; Katha ${ }^{1},{ }^{3}$

Kirby, Cat. Orth. III $33(1910)^{2}$, F. I. Bhamo ${ }^{1}{ }^{3}$ Orth. p. 65, f. $57(1914)^{3}$; Hanc., Rec. Sibsagar 4

Ind. Mus. XI $112(1915)^{4}$.

latipennis, Hanc., Rec. Ind. Mus. XI 113 Moradabad ${ }^{1}$ $(1915)^{1}$.

rotundatus, Hanc., Rec. Ind. Mus. XI Tezpur ${ }^{1}$ $112-113(1915)^{1}$.

\section{scaber, Thunberg}

Acrydium scabrum, Thnb., Nova Acta South India 58 Upsal, VII 159 (1815) ${ }^{1}$. Coimbatore ${ }^{10}$

Tettix subpustulata, Wlk., Cat. Derm. Palamcottah ${ }^{10}$ Salt. V $819(1871)^{2}$. Kezanathum ${ }^{10}$

Tettix scaber, Stāl, Recens. Orth. I 149 Bangalore ${ }^{10}$ $(1873)^{3}$. Nandyal ${ }^{10}$ 
Paratettix scaber, Bol., Ann. S. E. Belg. Annampallee ${ }^{10}$ XXXI 188, 279 (1887) ${ }^{4}$, Ann. S. E. Fr. LXX 585-586 (1902) ${ }^{5}$.

Paratettix scaber ugandensis, Karny, Sitz. Akad. Wiss. Wien. CXVI 26 (1907)

Paratettix scaber, Kirby, Cat. Orth. III $32(1910)^{7}$, F. I. Orth. p. $62(1914)^{8}$; Bol, Tr. Linn. Soc. (2) XV 267 (1912)9, Rev. R. Acad. Cienc. Madrid (2) XVI $287(1918)^{10}$.

semihirsutus, Brunn., Ann. Mus. Genova XXXIII 110-111 (1893)'; Kirby, Cat. Orth. III $33(1910)^{2}$, F. I. Orth. p. 66, f. $58(1914)^{3}$; Hanc., Rec. Ind. Mus. XI $113(1915)^{4}$.

34. Acrydium, Geoffroy (1962).

Type : subulatum, Linn. [Europe, W. and N. Asia]

Acrydium, Geoff., Hist. Ins. I 390 (1762) Fabr., Syst. Entom. p. 278 (1775); Rossi, Faun. Etrusc. p. 262 (1790); Oliv., Enc. Méthod. Ins. VI 209 (1791) ; Leach, Edinb. Encycl. IX 120 (1815) ; Curt., Brit. Ent. X t. 439 (1833) ; Steph., Mll. Brit. Ent., Mandib. VII 32 (1835); Kirby, Cat. Orth. III 35 (1910), F. I. Orth. pp. 66-67 (1914); Hanc., Rec. Ind. Mus. XI 60 [charact.], 114 (1915).

Bulla, Schrank, Enum. Ins. Austr. p. 242 (1781).

Acridium, Schrank, Fauna Boica II 30 (1801) ; Zett., Orth. Suec. p. 104 (1821), Faun. Ins. Lapp. I 452 (1828); Phil., Orthopt. Berol. p. 40 (1830); Zett., Ins. Lapp. p. 250 (1840).

Tetrix, Latr., Hist. Nat. Crust. Ins. III 284 Type : subulata, Linn. (1802), XII 161 (1804); St. Farg. and Serv., Encycl. Meth. Ins. X 598 (1825); Serv., Ann. Sci. Nat. XXII

South Africa ${ }^{2}, 3$, ?

Zanzibar ${ }^{4}, 8$

East Africa ${ }^{8}$

Gaboon ${ }^{4}, 8$

Aldabra ${ }^{9}$

Seychelles ${ }^{9}$

Karen Hills ${ }^{1}, 3$

Type : subulatum, Linn.

Type $: . . . .$. ?

Type $: \ldots \ldots$ ?...... 
264, 291 (1831), Ins. Orthopt. p. 757 (1839); Wesm., Bull. Acad. Brux. V 596 (1838) ; Burm., Handb. Ent. II 657 (1838) ; Borck, Skand. Rätv. Ins. p. 138 (1848); Finot. Orth. France pp. 39, 82 (1883), Faune France Orth. pp. 99, 165 (1889); Jacobs. and Bianchi, Prem. i Lozhn. Ross. Imp. pp. 174, 208 (1902) ; Hanc., Tett. N. Amer. pp. 35, 38, 66, 68 (1902), Spol. Zeyl. II 108, 110, 141 (1904) [redescr.], Gen. Ins. Orth. pp. 52, 57 (1906) [charact.], Ind. Agric. Ent. Mem. IV 149 (1912) ; Lucas, Brit. Orth. p. 206 (1920).

Tettix, Charp., Germ. Zeitschr. Ent. III 315 Type: subulatus, Linn. (1841) ; Fieb., Abh. Böhn. Ges. (5) III 408 (1844); Fisch. Waldh., Orth. Russ. p. 347 (1846); Fisch., Orth. Eur. p. 215 (1853); Fieber, Lotos III 139 (1853), Syn. Eur. Orth. p. 29 (1854) ; Türk, Wien. Ent. Mon. VI 206 (1862); Friv., Ertek. Termész. Köreb. I (12) p. 188 (1868); Stâl, Recens. Orth. I 146 (1873); Bol., Ortopt. Esp. p. 161 (1876), An. Soc. Esp. V 367 (1876); Brunn., Prodr. Eur. Orth. pp. 87, 234 (1882) ; Bol., Ann. S. E. Belg. XXXI 187, 195, 257 (1887), Ann. Sci. Nat. Porto IV 221, V 35 (1897-98); Burr, Brit. Orth. pp. 33, 46 (1897) ; Auriv., Ent.

Tidskr. XXI 241, 247 (1900); Tümpel, Geradfl. Mitteleuropas pp. 220, 253 (1900) ; Hanc., Spol. Zeyl. II 141142 (1904) [redescr.].

\section{bipunctatum, Linn.}

Gryllus Bulla bipunctatus, Linn., Syst. Kodaikanal14, ${ }^{17}$

Nat. (ed. X) p. $427(1758)^{1}$, Sulz., Sikkim ${ }^{18}$

Gesch. Ins. p. 82 , t. 8 f. $6(1776)^{2}$. 
Acrydium scutellatum, de Geer, Mém.

Ins. III 483 , t. 23 f. $15(1773)^{3}$.

Acrydium bipunctatum, Herbst, Fuess.

Arch. p. 189, t. 52 f. $1(1786)^{4}$.

Acrydium opacum, Herbst, Fuess. Arch.

p. 190 , t. 52 f. $2(1786)^{5}$.

Acrydium gibbum, Oliv., Enc. Meth. Ins. Persian Gulf ${ }^{18}$

VI $233(1791)^{6}$.

Tetrix bipunctata, Serv., Ins. Orthopt. N. \& W. Asia ${ }^{16}$

p. $765(1839)^{?}$.

Tettix schrankii, Fieb., Abhandl. Böhm. N. Africa ${ }^{16}$

Ges. (5) III 412, t. 10 ff. 17-19 (1843) .

Tellix bipunctatus, Brunn., Prodr. Eur.

Orth. pp. 234, $235(1882)^{9}$; Bol., Ann. Europe ${ }^{16},{ }^{18},{ }^{19}$

S. E. Belg. XXXI $263(1887)^{10}$; Burr.

Brit. Orth. p. 46 , t. 4 f. $2(1897)^{11}$;

Tümpel, Geradfl. Mitteleuropas pp. 220 , 253, t. $20(1900)^{12}$.

Tetrix bipunctata, Jacobs. and Bianchi,

Prem. i Lozhn. Ross. Imp. pp. 174, 209, t. $7(1902)^{13}$.

Tettix bipunctatus, Bol., Ann. S. E. Fr. LXX 585 (1902) $^{14}$.

Tetrix bipunctata, Hanc., T. E. S. 1910 $359(1910)^{15}$.

Acrydium bipunctatum, Kirby, Cat. Orth.

III 39-42 (1910) [numerous other refs. and synonyms] $]^{16}$, F. I. Orth. pp. 6869, ff. 59, 60 (1914) ${ }^{17}$; Hanc., Rec. Ind.

Mus. XI $114(1915)^{18}$.

Tetrix bipunctatus, Lucas Brit. Orth. pp. $212-217$, t. 21 f. $1(1920)^{19}$

\section{ceylonum, Hanc.}

Tettix atypicalis ceylonus, Hanc, Spol. Pundaluoya1, 2, 4

Zeyl. II 108, 142, 143-144 (1904)1. ${ }^{1}$ Maskeliya ${ }^{1},{ }^{4}$

Tetrix atypicalis ceylonus, Hanc., T. E. S. Peradeniya ${ }^{5}$

$1907239(1907)^{2}$.

Acrydium ceylonicum, Kirby, Cat. Orth.

III $45(1910)^{3}$.

Acrydium atypicale ceylonum, Kirby, F.

I. Orth. pp. 67-68 (1914) . 
Acrydium ceylonicum, Hanc., Rec. Ind. Mus. XI 115 (1915) [short-wing form of variegatum, Bol. $]^{5}$.

\section{cingalense, Wlk.}

Tettix cingalensis, Wlk., Cat. Derm. Ceylon ${ }^{1}, 2,8,12,11$

Salt. V 827 (1871) 1 . Madulsima ${ }^{10}$

Paratettix variegatus, Bol., Ann. S. E. Kandy ${ }^{4},{ }^{13}$

Belg. XXXI 188, 272, $280(1887)^{2}$; Peradeniya ${ }^{4},{ }^{13},{ }^{14}$

Hanc., Spol. Zeyl. II 108, 144-145 Pundaluoya ${ }^{5}$ $(1904)^{3}$.

Dikoya ${ }^{4},{ }^{13}$

Tettix atypicalis, Hanc., Spol. Zeyl. II - Ratnapura ${ }^{14}$

108, 142-143, t. 3 ff. 14, $14 a, b(1904)^{4}$. Pondichery ${ }^{11}$

Tetrix atypicalis, Hanc., T. E. S. 1907 Taliparamba ${ }^{15}$

$239(1907)^{5}$.

(Malabar)

Tetrix variegatus, Hanc., T. E. S. 1908 Singla (Darjiling distr.;

$413(1909)^{6}$.

Euparatettix cingalensis, Kirby, Cat. Moulmein ${ }^{14}$

Orth. III $31(1910)^{?}$.

Paratettix variegatus, Kirby, Cat. Orth. Java ${ }^{6}$

III $33(1910)^{8}$.

Acrydium atypicalis, Kirby, Cat. Orth.

III $45(1910)^{9}$.

Euparatettix variegatus, Hanc., Spol.

Zeyl. VI $148(1910)^{10}$,

Tetrix variegatus, Hanc., Ind. Agric.

Ent. Mem. IV 149 (1912) ${ }^{11}$.

Paratettix cingalensis, Kirby, F. I. Orth.

pp. 63-64, f. 56 (1914) ${ }^{12}$.

Acrydium atypicale, Kirby, F. I. Orth.

p. $67(1914)^{13}$.

Acrydium variegatum, Hanc., Rec. Ind.

Mus. XI 114-115 (1915) ${ }^{14}$.

Paratettix cingalensis, Bol., Rev. R. Acad. Cienc. Madrid (2) XVI 287 $(1918)^{15}$.

\section{discale, Wlk.}

Tettix discalis, Wlk., Cat. Derm. Salt. V Ceylon ${ }^{\mathbf{1},{ }^{2}}$ $826(1871)^{1}$.

Acrydium discalis, Kirby, Cat. Orth. III $45(1910)^{2}$. 


\section{indicum, Bol.}

Paratettix indicus, Bol., Ann. S. E. Belg. "East Indies"1 XXXI 188, 272, $281(1887)^{1}$, Ann. S. E. South India ${ }^{2}{ }^{5}$ Fr. LXX 585 (1902) $^{2}$; Kirby, Cat. Coimbatore ${ }^{6}$ Orth. III 33 (1910) $^{3}$; Hanc., Ind. Bihar ${ }^{4}$, Agric. Ent. Mem. IV 150 (1912) ${ }^{4}$; Kirby, F. I. Orth. pp. 64-65 (1914) $)^{5}$ China ${ }^{1-8 ;} 5$ Bol., Rev. R. Acad. Cienc. Madrid (2) XVI $288(1916)^{6}$.

Acrydium indicum, Hanc., Rec. Ind. Mus. XI 115 (1915)?

\section{indum, Olivier.}

Acrydium indum, Oliv., Enc. Méth. Ins. "East Inảics", 2 ". VI 234 (1791)

Acrydium? indum, Kirby, Cat. Orth. III $49(1910)^{2}$.

Acrydium indicum, Kirby, F. I. Orth. p. 77 (1914) $^{3}$; Bol., Rev. R. Acad. Cienc. Madrid (2) XVI $288(1918)^{4}$.

\section{lineosum, Wlk.}

Tettix lineosa, Wlk., Cat. Derm. Salt. V, Bombay ${ }^{1,2}$ Suppl. p. $90(1871)^{1}$.

Acrydium lineosum, Kirby, Cat. Orth. III $45(1910)^{2}$.

mundum, Wlk., Cat. Derm. Salt. V 823- North India1, 2 824 (1871) [Tettix munda] $]^{1}$; Kirby, Cat. Orth. III 45 (1910) $^{2}$; Hanc., Rec. Ind. Mus. XI $115(1915)^{3}$.

nigricolle, Wlk., Cat. Derm. Salt. V 826 Bombay 1, 2 (1871) [Tettix nigricollis] ${ }^{1}$; Kirby, Cat. Orth. III $45(1910)^{2}$.

obliquiferum, Wlk., Cat. Derm. Salt. V Bombay 1, " 825 (1871) [Tettix obliquifera] ${ }^{1}$; Kirby, Cat. Orth. III $45(1910)^{2}$.

\section{quadriplagiatum, Wlk.,}

Tettix quadriplagiata, Wlk., Cat. Derm. Nortb Iudiat-3 Salt. V $828(1871)^{1}$. 
Hedotettix quadriplagiata, Kirby, Cat. Orth. III $49(1910)^{2}$.

Acrydium quadriplagiatum, Kirby, F. I. Orth. p. 69 , f. $61(1914)^{3}$.

tectitergum, Hanc., Rec. Ind. Mus. XI Hoshangabad $115-116(1915)^{1}$.

Surat ${ }^{1}$.

umbriferum, Wlk., Cat. Derm. Salt. V 824 Bombay ${ }^{1},{ }^{2}$

(1871) [Tettix umbrifera]'; Kirby, Cat.

Orth. III $45(1910)^{2}$.

vittiferum, Wlk., Cat. Derm. Salt. V Bombay ${ }^{1},{ }^{2}$ 824-825 (1871) [Tettix vittifera] $]^{1}$; Kirby Cat. Orth. III $45(1910)^{2}$.

35. Coptotettix, Bolivar (188\%).

Type: asperatus, Bol. [Cochin China]. Bol., Ann. S. E. Belg. XXXI 188, 195, 287

(1887) ; Hanc., Spol. Zeyl. II 108, 111, 152 (1904) [redescr.], Gen. Ins. Tetrig. pp. 52, 65 (1906) [charact.]; Kirby, Cat. Orth. III 55 (1910), F. I. Orth. p. 74 (1914) [redescr.]; Hanc., Rec. Ind. Mus. XI 60 [charact.], 116-118 [key to spp.] (1915).

annandalei, Hanc., Rec. Ind. Mus. XI 119 $(1915)^{1}$.

Singla (Darjiling distr. ; $1,500$ feet $)^{1}$

Calcutta ${ }^{1}$

N. Shan Hills ${ }^{1}$

artolobus, Hanc., Rec. Ind. Mus. XI 121 Ceylon $^{1}$ $(1915)^{1}$.

capitatus, Bol., Ann. S. E. Belg. XXXI Karen Hills ${ }^{2-4}$ 188, 287, 289 (1887)1; Brunna., Ann. Somwarpet (Coorg) $)^{6}$

Mus. Genova XXXIII $111(1893)^{2}$;

Kirby, Cat. Orth. III $55(1910)^{3}$, F. I. Java ${ }^{1}{ }^{3}$

Orth. pp. 76-77 (1914) ${ }^{4}$; Hanc., Rec.

Ind. Mus. XI 118 (1915)5; Bol., Rev.

R. Acad. Cienc. Madrid (2) XVI 288 $(1918)^{6}$.

conspersus, Hanc., Rec. Ind. Mus. XI Sibsagar ${ }^{1}$ $119-120(1915)^{1}$

Siliguri ${ }^{1}$

Bhim Tal ${ }^{1}$ 
fossulatus, Bol., Ann. S. E. Belg. XXXI Ceylon ${ }^{1-4}$ 188, 287, 288-289 (1887) ${ }^{\mathbf{1}}$; Hanc., Puri distr. ${ }^{5}$ Spol. Zeyl. II 108, 153 (1904)2; Kirby, Cat. Orth. III $55(1910)^{3}$, F.I. Orth. p. 76-(1914)4; Hanc., Rec. Ind. Mus. XI $118(1915) .^{5}$

indicus, Hanc., Ind. Agric. Ent. Mem. IV Kalyan ${ }^{1}$ 144-145 (1912)1, Rec. Ind. Mus. VIII Satara distr. ${ }^{3}$ 313 (1913) [female descr.] ${ }^{2}$, Rec. Ind. Aborland ${ }^{3}$ Mus. XI $120(1915)^{3}$.

interruptus, Bol., Ann. S. E. Belg. XXXI Rangoon ${ }^{2}, 4$ 189, 288, 291-292 (1887)1; Brunn., Ann. Bhamo2 , 4 Mus. Genova XXXIII $113(1893)^{2}$; Karen Hills ${ }^{2}{ }^{4}$ Kirby, Cat. Orth. III $56(1910)^{3}$, F. I. Orth. p. 78 (1914) ${ }^{4}$; Hanc., Rec. Ind. Java ${ }^{1}{ }^{3}, 4$ Mus. XI $118(1915)^{5}$.

parvulus, Hanc., Ind. Agric. Ent. Mem. Chapra1 ${ }^{1} 3$

IV 145-146 $(1912)^{1}$, Rec. Ind. Mus. Calcutta ${ }^{3}$

VIII $314(1913)^{2}$, Rec. Ind. Mus. XI Kushtea (Bengal) ${ }^{3}$ $118(1915)^{3}$.

Singla (Darjiling distr.; $1,500 \mathrm{feet}^{3}$

Sikkim $^{3}$

Janakmukh ${ }^{2}$

$\mathrm{Kobo}^{2}$

Dibrugarh $^{2}$

retractus, Hanc., Rec. Ind. Mus. XI 120- Pusa ${ }^{1}$ $121(1915)^{1}$.

testaceus, Bol., Ann. S. E. Belg. XXXI Ceylon ${ }^{1-4}$

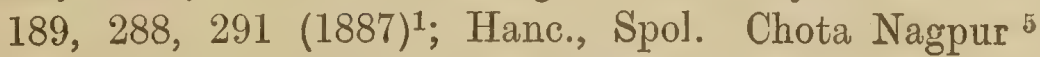
Zeyl. II 108, 153-154 (1904)2; Kirby, Cat. Orth. III $56(1910)^{3}$, F. I. Orth. p. $77(1914)^{4}$; Hanc., Rec. Ind. Mus. XI $121(1915)^{5}$.

\section{Hedotettix, Bolivar (188\%).}

Type : gracilis, de Haan.

Bol., Ann. S. E. Belg. XXXI 188, 195, 283 (1887); Hanc., Spol. Zeyl. II 108, 111, 148 (1904) [redescr.], Gen. Ins. Tetrig. pp. 52, 60 (1906) [charact.]; Kirby, Cat. Orth. III 49 (1910), F. I. Orth. p. 71 (1914) 
[redescr.]; Hanc., Rec. Ind. Mus. XI 60

[charact.], 121-122 [key to spp.] (1915).

attenuatus, Hanc.s Spol. Zeyl. II 108, Colombo1, 4, 5

149, 151-152, t. 3 ff. 18, $18 a, b(1904)^{1}$, Trincomali ${ }^{5}$

Gen. Ins. Tetrig. p. 60, f. $23(1906)^{2}$; Puri $^{5}$

Kirby, Cat. Orth. III $50(1910)^{3}$, F. I. Sibsagar ${ }^{5}$

Orth. p. 73 (1914) ${ }^{4}$; Hanc., Rec. Ind. Assam-Bhutan Frontier ${ }^{5}$ Mus. XI $123(1915)^{5}$.

cristitergus, Hanc., Rec. Ind. Mus. XI Hoshangabad ${ }^{1}$ $122(1915)^{1}$.

costatus, Hanc., Ind. Agric. Ent. Mem. Bengal ${ }^{1,2}$

IV 147-148 (1912) ${ }^{1}$, Rec. Ind. Mus. Bihar ${ }^{1}{ }^{2}$

XI $123(1915)^{2}$.

Nepal ${ }^{2}$

Allahabad ${ }^{2}$

\section{gracilis, de Haan.}

Acridium ('Tetrix) gracile, de Haan, Throughout. India (to Temm., Verh., Orth. pp. 167, 169 Peshawar), Burma and $(1842)^{1}$.

Hedotettix gracilis, Bol., Ann. S. F. Belg. XXXI 188, 283, $284(1887)^{2}$.

Heditettix festivus, Bol., Ann. S. E. Belg. XXXI 188, 284, 286, t. 5 ff. 24, .24a $(1887)^{3}$.

Ceylon.

Hedotettix gracilis, Brunn., Ann. Mus.

Genova XXXIII 111 (1893); Bol., Ann. S. E. Fr. LXX 586 (1902) [variation $]^{5}$; Hanc., Spol. Zeyl. II 108, 149151, t. 3 ff. 19, $19 a, b .(1904)^{6}$.

Hedotettix gracilis abortus, Hanc., Spol. Zeyl. II 108, 149, 151 (1904)?

Hedotettix gracilos, Hanc., T. E. S. 1907 $240(1907)^{8}$, T. E. S. $1908422(1909)^{9}$. Hedotettix gracilis abortus, Hanc., T. E. S. $1908422(1909)^{10}$.

Hedotettix gracilis, Kirby, Cat. Orth. III 49-50 (1910)11, Hanc., Spol. Zeyl. VI 149 (1910) [habits] ${ }^{12}$, Ind. Agric. Ent. Mem. IV $147(1912)^{13}$.

Hedotettix diminutus, Hanc., Ind. Agric. Ent. Mem. IV 148-149 (1912) ${ }^{14}$. 
Hedotettix gracilis, Kirby, F. I. Orth.

p. 72, ff. 64, 65 (1914) $)^{15}$; Hanc., Rec. Ind. Mus. XI $123(1915)^{16}$; Bol., Rev. R. Acad. Cienc. Madrid (2) XVI $288(1918)^{17}$.

grossus, Hanc., Rec. Ind. Mus. XI 124 Singla (Darjiling distr.; $(1915)^{1}$. $1,500$ feet $)^{1}$

lineifer, Wlk.

Tettix lineifera, Wlk., Cat. Derm. Salt. V Bombay ${ }^{1-3}$ $824(1871)^{1}$.

Acrydium lineiferum, Kirby, Cat. Orth. III $45(1910)^{2}$.

Hedotetlix? lineiferum, Kirby, F. I. Orth. p. 73 , f. $66(1914)^{3}$.

\section{Euparatettix, Hancock (1904).}

Type: personatus, Bol.

Euparatettix, Hanc., Spol. Zeyl. II 108, 111, Type: personatus, Bol. 145 (1904), Gen. Ins. Tetrig. pp. 51, 55 (1906) [charact.]; Kirby, Cat.

Orth. III 31 (1910); Hanc., Ind. Agric. Ent. Mem. IV 150-151 (1912) [key to spp.]; Kirby, F. I. Orth.p. 57 (1914) [redescr.]; Hanc., Rec. Ind. Mus. XI 60 [charact.], 124-125 [key to spp.] (1915).

Ergatettix, Kirby, F. I. Orth.pp.69-70 (1914). Type: scabripes, Bol. (=tarsalis, $\mathrm{Kby}$.)

\section{balteatus, Wlk.}

Tettix balteata, Wlk., Cat. Derm. Salt. V South India ${ }^{1-3}$ 828-829 (1871) 1 . Salem distr. ${ }^{4}$

Acrydium balteatum, Kirby, Cat. Orth. Palur ${ }^{4}$ III $45(1910)^{2}$.

Paratettix balteatus, Kirby, F. I. Orth. pp. 61-62, f. $54(1914)^{3}$.

Euparatetlix balteatus, Bol., Rev. R. Acad. Cienc. Madrid (2) XVI 286 $(1918)^{4}$. 
corpulentus, Hanc., Ind. Agric. Ent. Throughout India (to Mem. IV 153 (1912), Rec. Ind. Mus. Peshawar), Burma, XI $126(1915)^{2}$. and Ceylon.

\section{personatus, Bol.}

Paratettix personatus, Bol., Ann. S. E. Throughout India (to

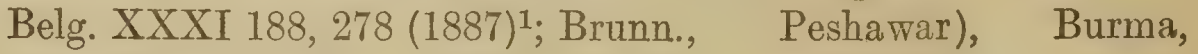
Ann. Mus. Genova XXXIII 109 and Ceylon. $(1893)^{2}$.

Euparatettix personatus, Hanc., Spol. Zeyl. II 108, 145, 146-148, t. 2 ff. 10, $10 a, b$, t. 3 ff. 20, 20a, b. (1904) $)^{3}$, Gen. Ins. Tetrig. p. 55, t. 3 f. $32(1906)^{4}$, T. E. S. $1907238(1907)^{5}$, T. E. S. 1908409 (1909) $^{6}$, Spol. Zeyl. VI 148149 (1910) [habits]; Kirby, Cat. Orth. III 31 (1910); $)^{8}$ Hanc., Ind. Agric. Ent. Mem. IV 152 (1912)'; Kirby, F. I. Orth p. 58, ff. 52, $53(1914)^{10}$; Hanc., Rec. Ind. Mus. XI $125(1915)^{11}$.

personatus birmanicus, Hanc., Rec. Rangoon ${ }^{1}$ Ind. Mus. XI 125-126 (1915) ${ }^{1} \quad$ Assam-Bhutan Frontier. ${ }^{1}$

\section{scabripes, Bol.}

Paratettix scabripes, Bol., Ann. Mus. South India ${ }^{2}$

Genova XXXIX $76(1898)^{1}$, Ann. Tinnevelly ${ }^{6}$

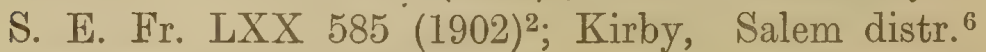

Cat. Orth. III $35(1910)^{3}$. Coimbatore ${ }^{6}$

Ergatettix tarsalis, Kirby, F. I. Orth. Taliparamba (Malabar) p. 70, ff. $62,63(1914)^{4}$.

Calcutta ${ }^{4}$

Euparatettix scabripes, Kirby, F. I. Rajshahi (Bengal $)^{5}$

Orth. p. 59 (1914) ${ }^{5}$; Bol., Rev. R. Acad.

Cienc. Madrid (2) XVI $286(1918)^{6}$.

tenuis, Hanc., Ind. Agric. Ent. Mem. IV 151-152 (1912) ${ }^{1}$, Rec. Ind. Mus. VIII $315(1913)^{2}$, Rec. Ind. Mus. XI 126 $(1915)^{3}$.

$\mathrm{Java}^{2},{ }^{3}$

Sumatra ${ }^{2}, 5$

Throughout United Prov., Bihar, Bengal and Assam.

Dawna Hills ${ }^{3}$

\section{variabilis, Bol.,}

Paratettix variabilis, Bol., Ann. S. E. Anuradhapura? Belg. XXXI 188, 271, 276-277 (1887) ${ }^{1}$, Madura ${ }^{1}$, ${ }^{4}, 5$ Ann. S. E. Fr. LXX 585 (1902); Puri ${ }^{7}$ 
Hanc., T. E. S. $1907236 \quad(1907)^{3}$; Asansol ${ }^{7}$

Kirby, Cat. Orth. III $33(1910)^{4}$, F. I. Berhampur?

Orth. pp. 62-63 (1914)

Rajshahi?

Euparatettix variabilis, Hanc., Ind. Agric. Rajmahal ${ }^{7}$

Ent. Mem. IV 150 (1912) $^{6}$, Rec. Ind. Pusa

Mus. XI $126(1915)^{7}$.

Shillong

Mandalay ${ }^{7}$

Borneo $^{1}, 3,5$

Philippines ${ }^{1},{ }^{4}, 5$

38. Indatettix, Hancock (1915).

Type : nodulosus, Hanc.

Hanc., Rec. Ind. Mus. XI 60 [charact.], 127 -

129 [key to spp.] (1915).

callosus, Hanc., Rec. Ind. Mus. XI 130 $(1915)^{1}$.

Singla (Darjiling distr. ; $1,500$ feet. $)^{1}$

crassipes, Hanc., Ind. Agric. Ent. Mem. Throughout United

IV 153-164 (1912) [Euparatettix] $]^{1}$, Prov., Bihar, Bengal

Rec. Ind. Mus. XI 130-131 (1915)². Gauhati.

crassipes bengalensis, Hanc., Ind. Agric. Pusa ${ }^{1}$

Ent. Mem. IV 154-155 (1912) [Eupara- Muzaffarpur ${ }^{1}$

tettix bengalensis $]^{1}$, Rec. Ind. Mus. XI Dacca ${ }^{1}$

$131(1915)^{2}$.

crassipes hybridus, Hanc., Rec. Ind. Dibrugarh ${ }^{1}$

Mus. XI $131(1915)^{1}$. Madhupur $^{1}$

Nepal ${ }^{1}$

crassipes var. A, Hanc., Rec. Ind. Mus. Chapra ${ }^{1}$

XI $131(1915)^{1}$. $\quad$ Pusa $^{1}$

interruptus, Brunn.,

Paratettix interruptus, Brunn., Ann. Mus. Karen Hills ${ }^{1}, 4$ Genova XXXIII 109-110 (1893) . Bhamo ${ }^{1}{ }^{4}$

Euparatettix interruptus, Hanc., T. E. S. Teinzo ${ }^{1,4}$ 1910 359-360 (1910) ${ }^{2}$; Kirby, Cat. Damukdia ${ }^{6}$

Orth. III $31(1910)^{3}$, F. I. Orth. p. 59 Madhupur ${ }^{6}$

[? f. 48 on p. 51] (1914) ${ }^{4}$; Bol., Rev. Calcutta ${ }^{6}$

R. Acad. Cienc. Madrid (2) XVI 59 Puri6

$(1918)^{5}$.

Coimbatore ${ }^{5}$ 
Indatettix interruptus, Hanc., Rec. Ind. Marikuppam ${ }^{6}$ Mus. XI $130(1915)^{6}$.

Travancore $^{6}$

Batticaloa (Ceylon) ${ }^{6}$

$\overline{\text { Johore }^{2}}$

interruptus var. A, Hanc., Rec. Ind. Satara distr. ${ }^{1}$ Mus. XI $130(1915)^{1}$.

interruptus lobulatus, Hanc., Rec. Ind. Hoshangabad ${ }^{1}$ Mus. XI 130 (1915) $^{\mathbf{1}}$.

nodulosus, Hanc., Ind. Agric. Ent. Mem. Throughout United IV 155-156 (1912) [Euparatettix] $]^{1}$, Prov., Bihar and BenRec. Ind. Mus. XI $129(1915)^{2}$. gal.

Garhwal² .

Nagpur

Satara ${ }^{2}$

\section{parvus, Hanc.}

Euparatettix parvus, Hanc., Spol. Zeyl. Elephant Pass (Ceylon) ${ }^{\mathbf{1}}{ }^{\mathbf{3}}$ II $108,145-146(1914)^{1}$.

Weligama (Ceylon) ${ }^{3}$

Euparatettix pilosus, Hanc., T. E. S. 1908 S. Malabar 409-410 (1909)², Spol. Zeyl. VI 149 Mysore 2. $(1910)^{3}$.

Central India ${ }^{2}$

Euparatettix parvus, Kirby, Cat. Orth. Puri ${ }^{8}$ III 31 (1910) ${ }^{4}$; Hanc., Ind. Agric. Ent. Calcutta ${ }^{8}$ Mem. IV 156 (1912)5, Rec. Ind. Mus. Chaumahani (Barisal) VIII 315 (1913) $^{6}$; Kirby, F. I. Orth. Igatpuri ${ }^{8}$ p. $60(1914)^{7}$.

Indatettix parvus, Hanc., Rec. Ind. Mus. XI $129(1915)^{8}$.

Amangarh (U. P.) $)^{8}$

Dhampur (U. P. $)^{8}$

Bhim Tal ${ }^{8}$

Peshawar

Dibrugarh 6

Aborland $^{6}$

Chapra ${ }^{5}$

Pusa $^{5}$

\section{BATRACHIDIANZE.}

Batrachidiini, Kirby, F. I. Orth. p. 78 (1914).

Batrachidince, Hanc., Rec. Ind. Mus. XI 60 [charact.], 131 (1915). 


\section{Saussurella, Bolivar. (188\%).}

Type : cucullifera, Wlk. (cornuta, Bol., nec de Haan.)

Bol., Ann. S. E. Belg., XXXI 189, 196, 303 (1887) ; Hanc., Gen. Ins. Tetrig. pp. 67, 71 (1906) ; Kirby, Cat. Orth. III 61 (1910); Hanc., Ind. Agric. Ent. Mem. IV 156-157 [key to spp.] (1912); Kirby, F. I. Orth. pp. 78-79 (1914) [redescr.]; Hanc., Rec. Ind. Mus. XI 60 [charact.], 131 (1915).

\section{cucullifera, -Wlk.}

Teltix cucullifera, Wlk, Cat. Derm. Salt. Pegu ${ }^{3}, 4,6,7$ V $829(1871)^{1}$.

Saussurella cornuta [nec de Haan], Bol., Ann. S. E. Belg. XXXI 189, 303 Amoy $^{1}, 5,6$ $(1887)^{2}$; Brunn., Ann. Mus. Genova Hongkong ${ }^{6}$ XXXIII 113, t. 5 f. 45 (1893) ${ }^{3}$; Kirby, Cat. Orth. III 61 (1910) [part.] ${ }^{4}$

Saussurella cucullifera, Kirby, Cat. Orth. III $61(1910)^{5}$, F. I. Orth. p. 79, f. $71(1914)^{6}$.

Saussurella brunneri, Hanc., Ind. Agric. Ent. Mem. IV $156(1912)^{7}$, Rec. Ind. Mus. XI 131-132 (1915) ${ }^{8}$.

curticornu, Hanc., Ind. Agric. Ent. Mem. Pusa ${ }^{1}$ IV 158-159 (1912) ${ }^{1}$, Rec. Ind. Mus. XI $131(1915)^{2}$.

decurva, Brunn., Ann. Mus. Genova Pegu' ${ }^{1}{ }^{3}$ XXXIII 113, t. 5 f. 46 (1893) $^{1}$; Kirby, Tenasserim ${ }^{4}$ Cat. Orth. III 61 $(1910)^{2}$, F. I. Orth. N. Lakhimpur ${ }^{4}$ pp. 79-80 (1914)3; Hanc., Rec. Ind. Mus. XI $131(1915)^{4}$.

indica, Hanc., Ind. Agric. Ent. Mem. IV Lebong ${ }^{1}{ }^{2}$ 157-158 (1912) $)^{1}$, Rec. Ind. Mus. XI $131(1915)^{2}$.

Total 169 species. 


\section{INDEX TO GENERA.}

(NotE.-The numbers refer to the numbers of the genera and not of the pages.)

Abbasia, 13

Acanthalobus, 19

Acridium (Acrydium)

Acrydium, 34

Amphibotettix, 10

Amphinotus, 26

Apterotettix, 25

Batrachidia (Paratettix)

Birmana, 2

Bolotettix, 15

Bulla (Acrydium)

Cladonotus, 6

Coptotettix, 35

Criotettix, 17

Deltonotus, 5

Ergatettix (Euparatettix)

Eugavialidium, 12

Euparatettix, 37

Eurymorphosus, 23

Fieberiana, 3

Gavialidium, 14

Gignotettix, 8

Hancockia, 22

Hedotettix, 36

Hyboella, 31
Indatettix, 38

Lamellitettix, 30

Loxilobus, 18

Mazarredia, 27

Mnema (Tettilobus)

Oxyphyllum, 4

Paratettix, 33

Plagiocephalus (Fieberiana)

Potua, 9

Saussurella, 39

Scelhymena (Scelimena)

Scelimena, 11

Scelymena (Scelimena)

Spadotettix, 24

Systolederus, 21

Teredorus, 32

Tetrix (Acrydium)

Tettilobus, 7

Tettitelum, 20

Tettix (Acrydium)

Thoradonta, 16

Tripetalocera, 1

Xistra, 28

Xistrella, 29 


\section{INDEX TO SPECIES.}

(Note.-Names in italics are treated as synonyms and should be looked up under the names given in roman letters. The generic name noted against each specific name is that of the genus in which it is placed in this Catalogue. The species in each genus are placed in alphabetical order and the position of the genus is shown in the Index to Genera.)

abortus, Hedotettix gracilis acuteterminatus, Hyboella acutus, Lamellitettix

\section{, Loxilobus}

æqualis, Criotettix

alatus, Paratettix

alligator, Gavialidium

angulatum, Eugavialidium

angulifrons, Hyboella

annandalei, Coptotettix

anomalus, Bolotettix

apiculata, Thoradonta

armatus, Bolotettix

armigera, Acantholobus bispinosus

artolobus, Coptotettix

assamus, Loxilobus

attenuatus, Hedotettix

atypicalis, Tettix (Acrydium cingalense)

balteatus, Euparatettix

bengalensis, Indatettix crassipes

bipunctatum, Acrydium

birmanicum, Eugavialidium

birmanicus, Euparatettix perșonatus

bispinosus, Acanthalobus

brunneri, Saussurella cucullifera

burri, Gignotettix

callosus, Indatettix

capitatus, Coptotettix

carmichaeli, Teredorus

Beylonicum, Acrydium ceylonum

ceylonum, Acrydium

cinereus, Systolederus

cingalense, Acrydium

conioptica, Hyboella

conspersus, Coptotettix

contracta, Scelimena

contracta, Scelimena uncinata

convergens, Mazarredia

cornuta, Saussurella cucullifera

corpulentus, Euparatettix

costatus, Hedotettix

crassipes, Indatettix

cristitergus, Hedotettix

cristulata, Mazarredia

crocodilus, Gavialidium

cucullifera, Saussurella

cuneatus, Acanthalobus curticornis, Acanthalobus

curt cornu, Saussurella

curtipennis, Paratettix

decurva, Saussurella

dilatata, Hyboella

diminutus, Hedotettix gracilis

discale, Acrydium

,, Eugavialidium

dohertyi, Criotettix

dorsifer, Paratettix

dromadaria, Xistrella

dubia, Mazarredia

, Xistra

exsertus, Bolotettix

extremus, Criotettix

fastiditus, Criotettix

feæ, Eugavialidium

ferruginea, Tripetalocera

festivus, Hedotettix gracilis

flavopictum, Eugavialidium

flavopictus, Criotettix

fletcheri, Lamellitettix

Spadotettix

fossulatus, Coptotettix

frontalis, Teradorus

gavialis, Scelimena

ghumtiana, Mazarredia

gibbiceps, Deltonotus

gibbum, Acrydium bipunctatum

gracilis, Birmana

,, Hedotettix

grandis, Criotettix

gravelyi, Criotettix

greeni, Systolederus

grossus, Hedotettix

hancocki, Loxilobus

harpago, Scelimena

hastulatum, Tettitelum

hirsutus, Paratettix

humbertianus, Cladonotus

hybridus, Indatettix crassipes:

inæqualis, Mazarredia

india, Eugavialidium

indica, Saussurella 
indicum, Acrydium

indicus, Coptotettix

,, Criotettix

indum, Acrydium

inermis, Bolotettix

inornatus, Acanthalobus

insignis, Mazarredia

insularis, Mazarredia

interruptus, Coptotettix

, Indatettix

kempi, Eugavialidium

laticeps, Mazarredia

latifrons, Hyboella

, Mazarredia

latilobus, Eurymorphopus

latipennis, Paratettix

latiramus, Cladonotus

latispina, Acanthalobus bispinosus

lativertex, Mazarredia

lineifer, Hedotettix

lineosum, Acrydium.

lobatus, Bolotettix

lobulatus, Indatettix interruptus

logani, Scelimena

lugubris, Mazarredia (Bolotettix lobatus)

maculatus, Criotettix indicus

maximus, Criotettix

miliarius, Acanthalobus

montanus, Criotettix

multidentatum, Eugavialidium

mundum, Acrydium

nigricolle, Acrydium

nodosa, Scelimena gavialis

nodulosus, Indatettix

nullipennis, Hyboella

obesa, Hyboella

obliquiferum, Acrydium

obscurus, Criotettix spinilobus

obtusus, Apterotettix

oculatus, Bolotettix

opacum, Acrydium bipunctatum

ophthalmica, Mazarredia

orientalis, Criotettix

pachymerus, Fieberiana

pallidus, Criotettix

pallitarsis, Acanthalobus bispinosus

parvispinus, Loxilobus

parvulus, Coptotettix

parvus, Indatettix

pelops, Tettilobus

pennatum, Oxyphyllum

perplexa, Mazarredia

personatus, Euparatettix

pictipes, Bolotettix

pilosus, Indatettix parvus

\author{
pluricarinatus, Lamellitettix \\ portentosa, Hancockia \\ problematica, Hyboella \\ provertex, Spadotettix \\ pygmæus, Amphinotus \\ quadratus, Bolotettix \\ quadriplagiatum, Acrydium \\ retractus, Coptotettix \\ rosaceus, Amphibotettix \\ rotundatus, Paratettix \\ rufescens, Acanthalobus \\ rugosus, Criotettix
}

rugosus, Loxilobus hancocki

sabulosa, Potua

saginatus, Acanthalobus inornatus

saussurei, Eugavialidium

scaber, Paratettix

scabripes, Euparatettix

schrankii, Acrydium bipunctatum

sculpta, Mazarredia

scutellatum, Acrydium bipunctatum

semihirsutus, Paratettix

sikkimensis, Mazarredia

singlaensis, Mazarredia

sinuata, Thoradonta

spiculoba, Thoradonta

spinata, Scelimena

spinifrons, Tettilobus

spinilobus, Criotettix

striatus, Loxilobus

stylata, Xistra

subcucullatus, Deltonotus

subpustulata, Paratettix scaber

subserrata, Abbasia

subulatus, Loxilobus

tarsalis, Euparatettix scabripes

tectiformis, Deltonotus subcucullatus

tectitergum, Acrydium

tentata, Hyboella

tenuis, Euparatettix

testaceus, Coptotettix

triangularis, Bolotettix

tricarinatus, Criotettix

tumida, Hyboella

turrifer, Cladonotus

ugandensis, Paratettix scaber

umbriferum, Acrydium

uncinata, Scelimena

variabilis, Euparatettix

variegatus, Paratettix (Acrydium cingalense)

vidali, Loxilobus

vittiferum, Acrydium 